\title{
Holomorphic isometry from a Kähler manifold into a product of complex projective manifolds
}

\author{
Xiaojun Huang*1 and Yuan Yuan ${ }^{\dagger}$
}

\begin{abstract}
We study the global property of local holomorphic isometric mappings from a class of Kähler manifolds into a product of projective algebraic manifolds with induced FubiniStudy metrics, where isometric factors are allowed to be negative.
\end{abstract}

\section{Introduction}

In this paper, we study the global property for local holomorphic isometric mappings, up to isometric factors that are allowed even to be negative, from a Kähler manifold into a Cartesian product of projective algebraic manifolds equipped with the induced Fubini-Study metrics. Investigations of this kind started with a paper of Calabi, who first studied the global extension and Bonnet type rigidity of a local holomorphic isometric embedding from complex manifolds with real analytic Kähler metrics [C] into complex space forms. Afterwards, there appeared many studies along these lines of research. (see [U] [DL], for instance). In 2003, motivated from problems in algebraic number theory, Clozel-Ullmo [CU] were led to consider rigidity problems for local holomorphic isometries between bounded symmetric domains equipped with their Bergman metrics. More precisely, by reducing the problem to the rigidity problem for local holomorphic isometries, they proved an algebraic correspondence in the quotient of a bounded symmetric domain preserving the Bergman metric has to be a modular correspondence in the case of unit disc in the complex plane and bounded symmetric domains of rank $\geq 2$. More recently, Mok carried out a systematic study of this type of problems in a very general setting. Many important results have been obtained by Mok and Mok-Ng. (See [M2] [N1-2], in particular, the papers by Mok [M3] and Mok-Ng [MN], and the references therein). Mok in [M2-3] proved the total geodesy for a local holomorphic isometric embedding between bounded symmetric domains $D$ and $\Omega$ when either (i) the rank of each irreducible component of $D$ is at least two or (ii) $D=\mathbb{B}^{n}$ and $\Omega=\left(\mathbb{B}^{n}\right)^{p}$ for $n \geq 2$. Mok and $\mathrm{Ng}$ in [MN] proved the total geodesy when the map is a local volume preserving map, which in particular has applications to answer, in the affirmative, questions of Clozel-Ullmo in algebraic number theory. In a recent joint paper of the second author with Zhang [YZ], the total geodesy is obtained in the case of

\footnotetext{
${ }^{1}$ Supported in part by NSF-1101481.
} 
$D=\mathbb{B}^{n}$ and $\Omega=\mathbb{B}^{N_{1}} \times \cdots \times \mathbb{B}^{N_{p}}$ with $n \geq 2$ and $N_{l}$ arbitrary for $1 \leq l \leq p$. Earlier, $\mathrm{Ng}$ in [N2] had established similar result when $p=2$ and $2 \leq n \leq N_{1}, N_{2} \leq 2 n-1$.

When manifolds are Hermitian symmetric spaces of compact type, it is well known that the total geodesy for (local) holomorphic isometric embeddings is no longer the case, even for mappings between complex projective spaces equipped with the standard Fubini-Study metrics. For instance, the Veronese embedding from $\left(\mathbb{P}^{n}, \omega_{n}\right)$ into $\left(\mathbb{P}^{\frac{n(n+3)}{2}}, \omega_{\underline{n(n+3)}}\right)$ is an isometric embedding with conformal constant 2 , which is not linear. In this setting, the rigidity problem is to find out when the maps are holomorphic isometries up to isometric constants.

In this paper, we carry out a study of the rigidity problem for local holomorphic conformal maps into the product of complex projective manifolds with the induced Fubini-Study metrics. Our conformal factors are allowed to have mixed signs. This has immediate application to the case when the target manifold is the product of the Hermitian symmetric spaces of compact type equipped with the canonical metrics.

Geometrically, such a problem may be regarded as the question of understanding what modification of the canonical metric on the source manifold admits only rigid holomorphic isometric embeddings (up to conformal factors) into product of projective spaces. To state our main result, we let

$$
\left(M, \omega_{m}\right),\left(M_{1}, \omega_{M_{1}}\right), \cdots, \quad\left(M_{m}, \omega_{M_{m}}\right), \quad\left(M_{1}^{\prime}, \omega_{M_{1}^{\prime}}\right), \cdots, \quad\left(M_{v}^{\prime}, \omega_{M_{v}^{\prime}}\right)
$$

be Kähler manifolds. When the manifolds are irreducible Hermitian symmetric spaces of compact type equipped with canonical Kähler-Einstein metrics, we always normalize the canonical metrics to have the maximum holomorphic sectional curvature +2 as that for the projective space equipped with the Fubini-Study metric. Let $\left(\lambda_{1}, \cdots, \lambda_{v}\right)$ and $\left(\mu_{1}, \cdots, \mu_{m}\right)$ be two sets of positive real numbers. Let $U \subset M$ be a connected open subset and $G_{j}: U \rightarrow M_{j}^{\prime}$ be a non-constant holomorphic map for each $j$. We write $\omega_{M, G, \lambda}:=\omega_{M}+\sum_{j=1}^{v} \lambda_{j} G_{j}^{*} \omega_{M_{j}^{\prime}}$ for the modification of $\omega_{M}$ by $(G, \lambda):=\left(G_{1}, \cdots, G_{v} ; \lambda_{1}, \cdots, \lambda_{v}\right)$ over $U$. We are interested in the question: When are there holomorphic maps $F_{l}: U \rightarrow M_{l}$ for each $l \in\{1, \cdots, m\}$ such that $\omega_{M, G, \lambda}=\sum_{l=1}^{m} \mu_{l} F_{l}^{*} \omega_{M_{l}}$ ? What is the rigidity phenomenon in this setting? There is a case where we may not get anything interesting, due to the obvious cancelation. In fact, as shown in Example 6.2, when we do not have the following number theoretic property:

$$
\operatorname{span}_{\mathbb{Q}_{0}^{+}}\left\{\lambda_{j}\right\}_{j=1}^{v} \cap \operatorname{span}_{\mathbb{Q}_{0}^{+}}\left\{\mu_{l}\right\}_{l=1}^{m}=\{0\}
$$

we can easily construct examples where we do not have any good rigidity and global extension properties. Here we write $\mathbb{Q}_{0}^{+}$for the set of non-negative rational numbers.

A main result of this paper is to provide the following rigidity theorem, under the the needed number theoretic condition (1.1) for the conformal factors.

Theorem 1.1 Let $h(z, \xi)=a_{0}+\sum_{|\alpha|,|\beta|>0} a_{\alpha \beta} z^{\alpha} \xi^{\beta}$ be an irreducible holomorphic polynomial over $\mathbb{C}^{2 n}$ for $n \geq 1$ with $h(z, \bar{z})>0$. Let $M$ be a simply connected $n$-dimensional complex 
manifold (not necessarily complete) with a real analytic Kähler metric $\omega_{M}$. Assume that there exists a holomorphic coordinate chart $(U \subset X, \phi)$ with $\phi(U)=V \ni 0$, a connected open subset in $\mathbb{C}^{n}$, such that $\left(\phi^{-1}\right)^{*} \omega_{M}=\sqrt{-1} \partial \bar{\partial} \log h(z, \bar{z})$. Let $M_{l}=\mathbb{P}^{N_{l}}, M_{j}^{\prime}=\mathbb{P}_{j}^{N_{j}^{\prime}}$ be complex projective spaces equipped with the Fubibi-Study metrics $\omega_{l}, \omega_{j}^{\prime}$, respectively, for $1 \leq l \leq m, 1 \leq j \leq v$. Suppose that $F_{l}: U \subset M \rightarrow \mathbb{P}^{N_{l}}, G_{j}: U \subset M \rightarrow \mathbb{P}^{N_{j}^{\prime}}$ are non-constant holomorphic maps over $U$ for each $l, j$. Assume that

$$
\omega_{M}=\sum_{l=1}^{m} \mu_{l} F_{l}^{*} \omega_{l}-\sum_{j=1}^{v} \lambda_{j} G_{j}^{*} \omega_{j}^{\prime} \text { over } U
$$

for $\mu_{l}, \lambda_{j} \in \mathbb{R}^{+}$which satisfy the property in (1.1). Then $F_{l}$ and $G_{j}$ extend to global holomorphic immersions $\tilde{F}_{l}: M \rightarrow \mathbb{P}^{N_{l}}$ and $\tilde{G}_{j}: M \rightarrow \mathbb{P}^{N_{j}^{\prime}}$, respectively. Moreover $\tilde{F}_{l}^{*} \omega_{l}=m_{l} \omega_{M}, \tilde{G}_{j}^{*} \omega_{j}^{\prime}=$ $n_{j} \omega_{M}$ with $m_{l}, n_{j} \in \mathbb{N}$ satisfying the identity:

$$
1=\sum_{l=1}^{m} \mu_{l} m_{l}-\sum_{j=1}^{v} \lambda_{j} n_{j}
$$

In Theorem 1.1, when $M$ is not simply connected, then one can still conclude that the maps extend along any path inside $M$ initiated from a point in $U$. Theorem 1.1 applies immediately when $\left(M, \omega_{M}\right)$ is the projective space equipped with the Fubini-Study metric. Indeed, the special case of Theorem 1.1 with $M$ being $\mathbb{P}^{1}$ can be applied with the minimal rational curve theory on Hermitian symmetric spaces of compact type to yield the following:

Theorem 1.2 Let $\left(M, \omega_{M}\right),\left(M_{l}, \omega_{M_{l}}\right),\left(M_{j}^{\prime}, \omega_{M_{j}^{\prime}}\right)$ be irreducible compact Hermitian symmetric spaces of compact type equipped with the canonical Kähler-Einstein metrics $\omega, \omega_{l}, \omega_{j}^{\prime}$ for $1 \leq l \leq$ $m, 1 \leq j \leq v$, respectively. Let $\mu_{l}, \lambda_{j}>0$ be a set of real numbers for $1 \leq l \leq m$ and $1 \leq j \leq v$, satisfying (1.1). Let $F_{l}: U \rightarrow M_{l}$ and $G_{j}: U \rightarrow M_{j}^{\prime}$ be non-constant holomorphic maps for each $l, j$ such that

$$
\omega_{M}=\sum_{l=1}^{m} \mu_{l} F_{l}^{*} \omega_{M_{l}}-\sum_{j=1}^{v} \lambda_{j} G_{j}^{*} \omega_{M_{j}^{\prime}} \text { over } U,
$$

where $U \subset M$ is a connected open subset. Then for any $j \in\{1, \cdots, v\}$ and $l \in\{1, \cdots, m\}, F_{l}$ and $G_{j}$ extend to global holomorphic embeddings $\tilde{F}_{l}: M \rightarrow M_{l}$ and $\tilde{G}_{j}: M \rightarrow M_{j}^{\prime}$, respectively. Moreover $\tilde{F}_{l}^{*} \omega_{M_{l}}=m_{l} \omega_{M}, \tilde{G}_{j}^{*} \omega_{M_{j}^{\prime}}=n_{j} \omega_{M}$ with $m_{l}, n_{j} \in \mathbb{N}$ satisfying the equation (1.3).

Notice that in Theorem 1.1, the extended maps may not be one to one, while in Theorem 1.2 , they all are embeddings. Also in these two theorems, when $v=0$, it is understood that there are no mappings $G_{j}$. As one sees in Example 6.2, the assumption (1.1) on the conformal factors in Theorem 1.1 and Theorem 1.2 is to avoid the cancelation that destroys good rigidities, which together with (1.3) is necessary and sufficient for Theorem 1.1 and Theorem 1.2 to hold. 
This phenomenon is similar to the study of CR mappings: Non-existence of negative conformal factors corresponds to the strongly pseudoconvex case, while the investigation for the case of conformal factors with a mixed sign resembles to the Levi non-degenerate situation with positive signature (see [Hu2], [BH], for instance).

Theorem 1.1 and Theorem 1.2 follow trivially from the Calabi theorem, when all conformal factors are positive and rational (or at least rational up to a common factor). In this setting, one can apply the Nakagawa-Takagi theorem, the Veronese and Segre embeddings, to immediately reduce them to the study a local holomorphic isometric embedding into a projective space equipped with a multiple of the Fubini-Study metric. Hence, the results follows immediately from the classical Calabi theorem in [C]. (See Mok [M4] and Yuan [Y].) However, by Calabi [p. 23, C] (see also Remark 6.1), when $\mu_{1} / \mu_{2}$ is not a rational number, then the Kähler manifold $\left(\mathbb{P}^{n}, \mu_{1} \omega_{n}\right) \times\left(\mathbb{P}^{m}, \mu_{2} \omega_{m}\right)$ can not be isometrically embedded into the Hilbert projective space equipped with any multiple of the Fubini-Study metric $\left(\mathbb{P}^{\infty}, \mu \omega_{\infty}\right)$. (Here, $\mu>0$ is a real number.)

In [C], Calabi also considered the existence problem for isometric embeddings between complex manifolds with indefinite Kähler metrics. Since in this setting, there is always a cancelation even inside the metric part itself, it seems hard to get any rigidity and global extension result.

A first main step in our proof of Theorems 1.1 is to obtain the Nash-algebraicity for both $F_{l}$ and $G_{j}$ under the assumption of (1.1). We then prove a single-valuedness for algebraic functions satisfying a certain transcendental equation by employing the monodromy argument and the Puiseux expansion for multi-valued algebraic functions. The last step is to use what is obtained in previous steps, as well as, the geometry of minimal rational curves over the source manifold, to derive Theorem 1.2.

The organization of the paper is as follows. In Section 2, after collecting the basic properties of algebraic functions, we prove a certain induction property and boundedness for algebraic functions satisfying a certain transcendental functional identity. In Section 3 and Section 4, we prove the algebraicity and single-valuedness, respectively, for germs of holomorphic functions satisfying a certain transcendental functional identity. At the end of the section 4, we give the proof of Theorem 1.1. In Sections 5, we apply the results derived in Section 3 and 4, to prove Theorem 1.2. We end up our paper with examples which show our number theoretic condition (1.1) on the conformal factors are more or less necessary and sufficient conditions for our results to hold.

Acknowledgement: The authors would like to express their gratitude to N. Mok and S.-C. $\mathrm{Ng}$ for many very valuable discussions related to this paper. The authors would like also to thank J.-M. Hwang for several helpful communications related to this work in January, 2011. 


\section{$2 \quad$ Algebraic functions}

In this section, we start by first recalling some basic properties for algebraic functions. Then we will prove several lemmas to be used in Section 3 and Section 4. Our basic tools are the monodromy argument and the Puiseux expansion for multi-valued algebraic functions.

A holomorphic function $f$ over $U \subset \mathbb{C}^{n}$ is called a holomorphic Nash-algebraic, or simply holomorphic algebraic function, if there is an irreducible polynomial $P(z, X)$ in $X$ with coefficients in polynomials of $z$ such that $P(z, f(z)) \equiv 0$ over $U$. $f$ extends to multi-valued a function, still denoted by $f$, over $\mathbb{C}^{n}$, some branches of which may blow up along a complex analytic variety of codimension one. $P(z, X)$ is called a minimum polynomial of $f$. All branches of $f$ are bounded over any compact subset in $\mathbb{C}^{n}$ if and only if the leading coefficient of $P(z, X)$ can be made to be 1 . For our purpose in this article, we are mainly concerned with algebraic functions whose minimum polynomials have leading coefficient 1.

Let $H$ be an algebraic function in $U \subset \mathbb{C}^{n}$ with its minimum polynomial of leading coefficient 1: $P(z, Y)=Y^{d}+a_{1}(z) Y^{d-1}+\cdots+a_{d}(z)$, where $d \geq 1, a_{1}, \cdots, a_{d}$ are polynomials in $z$ and $P(z, Y)$ is an irreducible polynomial in $(Z, Y)$. Then there are polynomials $A(z, Y), B(z, Y)$ and $p(z)$ such that

$$
A(z, Y) P(z ; Y)+B(z, Y) \frac{\partial P}{\partial Y}(z ; Y)=p(z)
$$

Let $E \subset \mathbb{C}^{n}$ be the affine algebraic hypersurface defined by $p(z)=0$. Notice that any point $z_{0}$ with $p\left(z_{0}\right) \neq 0$ is a regular point for the algebraic function $H$, namely, a point $z_{0}$ where for any $Y$ with $P\left(z_{0}, Y\right)=0$ it holds that $\frac{\partial P}{\partial Y}\left(z_{0}, Y\right) \neq 0$. Then any branch of $H(z)$ can be holomorphically continued along a curve $\gamma \subset \mathbb{C}^{n} \backslash E$. Let $E_{0} \subset E$ be an irreducible component of $E$. We say that $E_{0}$ is a branching variety of $H$ if for a generic smooth point $p_{0} \in E_{0}$ and a sufficiently small ball $B_{p_{0}}$ centered at $p_{0}, \pi^{-1}\left(B_{p_{0}} \backslash E_{0}\right)$ has less than $d$ pieces of connected components. Here, letting $W \subset \mathbb{C}^{n} \times \mathbb{C}$ be defined by $P(z, Y)=0$, then $\pi$ is a branched covering map from $W$ to $\mathbb{C}^{n}$ giving by the natural projection map. Since $p_{0} \in E$ is a smooth point, after a holomorphic change of coordinates, we assume that $p_{0}=0$ and $E_{0}$ is defined by $z_{n}=0$. Being generic, we mean that $p_{0}=0$ is not contained in any other component of $E$. Let $\gamma(t)=\left(0, \cdots, 0, \epsilon e^{2 \pi \sqrt{-1} t}\right)$ for $0 \leq t \leq 1$, with $0<\epsilon<<1$ sufficiently small. Then for a small ball $B_{0}$ centered at 0 , any loop in $B_{0} \backslash E_{0}$ is homotopic to $k \gamma$ with $k \in \mathbb{Z}$. Any simple loop homotopic to $\gamma$ is called a basic loop around $E_{0}$ near $p_{0}=0$. Now, write $p^{*}=(0, \cdots, 0, \epsilon)$. Then $E_{0}$ is a branching hypervariety for $H$ if and only if for some holomorphic branch $H_{1}$ of $H$ in a neighborhood of $p^{*}$, when we holomorphically continue $H_{1}$ along $\gamma$ one round, we will obtain another branch $H_{2}\left(\neq H_{1}\right)$ of $H$ near $p^{*}$. When $\frac{H_{2}}{H_{1}}=h_{12}$ is a constant, then we have some $d_{0} \in \mathbb{N}$, such that, $h_{12}^{d_{0}}=1$. The smallest such a $d_{0}$ is called a period. In this case, we call $E_{0}$ a simple cyclic branching hypervariety for $H$ with respect to the branch $H_{1}$. Apparently, the simple cyclicity of $H$ along $E_{0}$ is independent of the choice of the generic point $p_{0}$. Furthermore, if there does not exist a polynomial function $h$ and a natural number $n_{1}$ such that $H=h^{\frac{1}{n_{1}}}$, then there must be an $n_{2} \in \mathbb{N}$ such that $H^{n_{2}}$ has branching varieties, none of 
which is a simple cyclic branching variety with respect to any branch of $H^{n_{2}}$. We also recall the Puiseux expansion for branches obtained by continuing $H_{1}$ near $p_{0}=0$, which will be the basic tool for us to deal with multi-valued holomorphic functions:

$$
H_{1}=\sum_{i=0}^{\infty} a_{i}\left(z^{\prime}\right) z_{n}^{\frac{i}{N_{0}}}
$$

with $N_{0}(\geq 2) \in \mathbb{Z}$ and $a_{i}\left(z^{\prime}\right)$ being holomorphic near $0^{\prime}$. Here $z^{\prime}=\left(z_{1}, \cdots, z_{n-1}\right)$.

The following lemma is essential in the later induction argument.

Lemma 2.1 Let $U \subset \mathbb{C}^{n}$ be a connected open subset. Let $\vec{h}(z)=\left(h_{1}, \cdots, h_{k}\right)(z) \in \mathbb{C}^{k}$ be a row of irreducible distinct polynomials. Let $\vec{r} \in(\mathbb{R} \backslash\{0\})^{k}$. Let $H_{1}(z), \cdots, H_{k}(z)$ be non-constant holomorphic algebraic functions defined over $U$ such that for a certain branch of the power function $\left(H_{\alpha}\right)^{\mu_{\alpha}}$ for each $\alpha$, and for a certain constant $c_{0}$, we have

$$
\vec{h}(z)^{\vec{r}}=c_{0} \prod_{\alpha=1}^{k} H_{\alpha}(z)^{\mu_{\alpha}} \quad \text { on } \quad U^{\prime}
$$

for $\mu_{1}, \cdots, \mu_{k} \in \mathbb{R} \backslash\{0\}$. Here $U^{\prime} \subset U$ is a subdomain such that $\vec{h}(z)^{\vec{r}}=h_{1}^{r_{1}} \cdots h_{k}^{r_{k}}$ has a well-defined holomorphic branch. Assume all the branches of $H_{\alpha}$ 's can only have zero or points of indeterminancy in the variety defined by the union of of the zeros of $h_{j}$ for each $j$. If $H_{1}$ has an irreducible non-cyclic branching hypervariety with respect to a certain branch, then there exists $n_{1}^{+}, n_{2}, \cdots, n_{k} \in \mathbb{Z}$ with $n_{1}^{+}>0$, such that

$$
\mu_{1} n_{1}^{+}=\sum_{\alpha=2}^{k} \mu_{\alpha} n_{\alpha}
$$

Furthermore, we have

$$
\vec{h}(z)^{n_{1}^{+} \vec{r}}=c_{0}^{n_{1}^{+}} \prod_{\alpha=2}^{k}\left(H_{1}^{n_{\alpha}} H_{\alpha}^{n_{1}^{+}}\right)^{\mu_{\alpha}} .
$$

In the above lemma and the rest of the paper, for an algebraic function $\phi$, we define the zero set (points of indeterminancy, respectively) of $\phi$ to be that defined by the zeroth order term (the coefficient of the highest order term, respectively) in a minimal polynomial of $\phi$.

Proof of Lemma 2.1: Assume without loss of generality that 0 is in $U$ and is a regular point for $H_{\alpha}$ for each $\alpha$. Since $H_{1}$ has an irreducible non-cyclic branching hypervariety $E$ with respect to a certain branch of $H_{1}$. Let $p^{*}(\notin E)$ be very close to a generic smooth point $p_{0}^{*}$ of $E$. In what follows, we assume that $E$ is defined by the zero of $h_{i}(z)$ for a certain $i$ or a small neighborhood of $p_{0}^{*}$ does not cut any zero of $h_{\alpha}^{\prime} s$. Let $\gamma$ be a basic loop around $E$ near $p_{0}^{*}$ with $\gamma(0)=p^{*}$. Assume that $\gamma$ stays in a sufficiently small ball centered at $p_{0}^{*}$. Assume $\left(H_{1}\right)_{1}$ is 
a holomorphic branch of $H_{1}$ at $p^{*}$ and when we continue holomorphically $\left(H_{1}\right)_{1}$ along $\gamma$ one round, we get a new holomorphic branch $\left(H_{1}\right)_{2}$ near $p^{*}$ with $\chi_{11}=\frac{\left(H_{1}\right)_{2}}{\left(H_{1}\right)_{1}} \neq$ constant. Choose $\gamma_{0}$, a simple curve connecting 0 to $p^{*}$ (that does not cut the zero of any $h_{j}$ and the union of branching varieties of $H_{l}$ for each $l$ ) such that we get $\left(H_{1}\right)_{1}$ near $p^{*}$ when we continue $H_{1}$ along $\gamma_{0}$ from its original holomorphic value near 0 . We still have by the uniqueness of real analytic functions:

$$
\vec{h}^{\vec{r}}=c_{0}\left(H_{1}\right)_{1}^{\mu_{1}} \cdots\left(H_{k}\right)_{1}^{\mu_{k}} \text { near } p^{*} .
$$

Here $\left(H_{\alpha}\right)_{1}^{\mu_{\alpha}}$ is a certain branch of the multi-value functions $e^{\mu_{\alpha} \log \left(H_{\alpha}\right)_{1}}$ near $p^{*}$ for any $\alpha$ with $1 \leq \alpha \leq k$. Now we holomorphically continue (2.4) along $\gamma$ to arrive at

$$
\vec{h}^{\vec{r}}=c_{0}^{\prime}\left(H_{1}\right)_{2}^{\mu_{1}} \cdots\left(H_{k}\right)_{2}^{\mu_{k}} \text { near } p^{*},
$$

since $h_{i}^{r_{i}}$ is at most cyclically branching at $p$ for each $1 \leq i \leq k$. From (2.4) and (2.5), we get

$$
c_{1}=\chi_{11}^{\mu_{1}} \cdots \chi_{1 k}^{\mu_{k}} \quad \text { near } p^{*}
$$

with $c_{1}(\neq 0)$ a certain constant, reflecting how we choose the branches of the multi-valued functions. Hence

$$
\chi_{11}=c_{1}^{\prime} \chi_{12}^{-\mu_{2} / \mu_{1}} \cdots \chi_{1 k}^{-\mu_{k} / \mu_{1}} \text { near } p^{*} .
$$

Here, $c_{1}^{\prime}(\neq 0)$ is a certain constant. Since $\chi_{11}$ is not a constant, we can find a complex line $L$ with a linear coordinate $\xi$ such that $\tau=\chi_{11}(\xi) \neq$ constant. Here and in what follows, we will write $\chi_{11}(\xi)$ for $\left.\chi_{11}\right|_{L}$. Hence

$$
\tau=c_{1}^{\prime \prime}\left(\chi_{12} \circ \chi_{11}^{-1}(\tau)\right)^{-\mu_{2} / \mu_{1}} \cdots\left(\chi_{1 k} \circ \chi_{11}^{-1}(\tau)\right)^{-\mu_{k} / \mu_{1}} .
$$

Now, after a holomorphic continuation, we can assume that the above holds for certain branches near the origin. Since $\chi_{1 \alpha} \circ \chi_{11}^{-1}(\tau)$ is algebraic for $2 \leq \alpha \leq k$, we have the Puiseux expansion near 0:

$$
\chi_{1 \alpha} \circ \chi_{11}^{-1}(\tau)=\sum_{i \geq i_{\alpha}} a_{\alpha i} \tau^{i / N_{0}}, a_{\alpha i_{\alpha}} \neq 0
$$

Thus, we get (2.2) by comparing the exponent of the lowest degree term in the expansion in $\tau$. Taking $n_{1}^{+}$-th power in (2.1) and applying (2.2), we get (2.3).

In the following, for an open subset $U \in \mathbb{C}^{n}$, we write $\operatorname{conj}(U):=\{z, \bar{z} \in U\}$. The following is a key lemma for our proof of Theorem 4.1 .

Lemma 2.2 Let $U_{1}$ be a connected open subset in $\mathbb{C}^{n}$, and let $\phi(z, \bar{z})$ be real analytic in $(z, \bar{z})$ over $\mathbb{C}^{n}$ with $\phi(z, \bar{z})>0$ for any $z \in \mathbb{C}^{n}$. Assume that $\phi(z, \xi)$ is holomorphic and algebraic in $U_{1} \times \operatorname{conj}\left(U_{1}\right)$. Suppose that $F_{l}(z): U_{1} \subset \mathbb{C}^{n_{1}} \rightarrow \mathbb{C}^{N_{l}}, G_{j}(z): U_{1} \subset \mathbb{C}^{n_{1}} \rightarrow \mathbb{C}^{N_{j}^{\prime}}$ non-constant holomorphic algebraic maps. Write $\bar{F}(\xi):=\overline{F(\bar{\xi})}$ for $\xi \in \operatorname{conj}\left(U_{1}\right)$. Suppose they satisfy the following identity 


$$
\prod_{l}\left(1+F_{l}(z) \cdot \overline{F_{l}}(\xi)\right)^{\mu_{l}}=\phi(z, \xi) \cdot \prod_{j}\left(1+G_{j}(z) \cdot \overline{G_{j}}(\xi)\right)^{\nu_{j}} \quad \text { over } U_{1} \times \operatorname{conj}\left(U_{1}\right),
$$

where $\mu_{j}, \nu_{j}$ are positive real numbers satisfying (1.1). Then the following holds:

1. Each component of $F_{l}(z)$ and $G_{j}(z)$ has a minimal polynomial with leading coefficient 1. In particular, for any compact subset $K_{1} \subset \mathbb{C}^{n_{1}}$, there is a constant $C_{K_{1}}$ such that $\left|h^{*}\right| \leq C_{K_{1}}$ holds over $K_{1}$, for any branch $h^{*}$ of a function component $h$ from $G_{j}$ or $F_{l}$.

2. Any branch of $1+F_{l}(z) \cdot \overline{F_{l}}(\xi)$ and $1+G_{j}(z) \cdot \overline{G_{j}}(\xi)$ can only have zeros on the hypervariety consisting of the zeros and points of inderterminancy of $\phi(z, \xi)$. (When $\phi(z, \xi)$ is not a polynomial, as mention before, the zero set (the set of points of indeterminancy) of $\phi(z, \xi)$ is the set defined by the zeroth order term (the coefficient of the highest order term, respectively) in a minimal polynomial of $\phi(z, \xi)$ )

Proof of Lemma 2.2: For simplicity of notation, assume that the first component of $F_{1}$, denoted by $h_{0}(z)=F_{1,1}(z)$, has a minimial polynomial with leading coefficient $a(z) \neq$ constant. Let $a_{0}(z)$ be an irreducible factor of $a(z)$, and let $K_{0}(z)=a(z) h_{0}(z)$. Then $K_{0}$ is an algebraic function with a minimum polynomial of leading coefficient 1 . Write $\left\{h_{1}, \cdots, h_{k}\right\}$ for the other function components in $F$ and $G$ whose respective leading coefficients $a_{1}, \cdots, a_{k}$ have the prime factor $a_{0}(z)$. Define similarly $K_{\alpha}(z)=a_{\alpha}(z) h_{\alpha}(z)$ for $\alpha=1, \cdots, k$. Choose a point $p_{0}$ such that (i) $p_{0}$ is a smooth point of the zero set of $a_{0}(z)$, (ii) the leading coefficients of the rest components are not zero at $p_{0}$ and the other components of $a_{1}, \cdots, z_{k}$ are not zero near $p_{0}$, and (iii) the zero set of $a_{0}$ near $p_{0}$, which may or may not be a branching variety itself, does not cut any other branching variety of components of $F_{l}, G_{j}$. Assume $p_{0}=0$ to simplify the notation. After a local change of coordinates, we may assume that $a_{0}(z)=z_{n}$. Now, by the analytic extension we can assume (2.6) with $\xi=\bar{z}$ holds for $(z, \bar{z})$ near $p_{0}=0$ for certain branches of $F_{l}$ and $G_{j}$. For $F_{1,1}$, the branch is chosen so that it is unbounded near $p_{0}$. (From the relation formula between roots and coefficients, and using the irreducibility of minimal polynomials, we can see that not all branches of $F_{1,1}$ can be bounded near $p_{0}$. Hence, we can pick the unbounded one to fit our consideration here.) Consider the Puiseux expansion of the corresponding branch $K_{\alpha}$ near $p_{0}$ for $0 \leq \alpha \leq k$, which we still write as $K_{\alpha}$ to simplify the notation:

$$
K_{\alpha}=\sum_{i=i_{\alpha} \geq 0}^{\infty} b_{\alpha, i}\left(z^{\prime}\right) z_{n}^{\frac{i}{n_{\alpha}}}
$$

with $b_{\alpha, i_{\alpha}}\left(z^{\prime}\right) \not \equiv 0$. Then

$$
\left|h_{\alpha}\right|^{2}=\left|z_{n}\right|^{2\left(\frac{i_{\alpha}}{n_{\alpha}}-k_{\alpha}\right)}\left|b_{\alpha, i_{\alpha}}\left(z^{\prime}\right)\right|^{2}+o\left(\left|z_{n}\right|^{2\left(\frac{i_{\alpha}}{n_{\alpha}}-k_{\alpha}\right)}\right),
$$

with $k_{\alpha}$ a certain natural number. 
We easily see that there exist rational numbers $r_{l} \leq 0, r_{j}^{\prime} \geq 0$ such that

$$
\left|z_{n}\right|^{\left(\sum_{l} r_{l} \mu_{l}+\sum_{j} r_{j}^{\prime} \lambda_{j}\right)} R(z, \bar{z})=\phi(z, \bar{z})
$$

near $p_{0}=0$. Notice that $r_{1} \leq 2\left(\frac{i_{1}}{n_{1}}-k_{1}\right)<0$, for, as we mentioned, the branch $h_{0}$ of $F_{1,1}$ is chosen to be unbounded near $p_{0}$. (Similarly, we have $r_{\alpha} \leq 0$ when the associate component is from $H^{\prime}$ 's and $r_{\alpha}^{\prime} \geq 0$ when the associate component is from $G$ 's.) Here for a generic fixed choice of $z^{\prime}$ near 0 , when $z_{n} \rightarrow 0,|R(z, \bar{z})|$ and $|R(z, \bar{z})|^{-1}$ remain to be bounded. Hence, we have $\sum_{l} r_{l} \mu_{l}+\sum_{j} r_{j}^{\prime} \lambda_{j}=0$, contradicting the hypothesis in (1.1). Thus Lemma 2.2 (1) is proved.

To prove the second part of the lemma, suppose that $p_{0}$ is not a zero nor a point of indeterminancy of $\phi(z, \xi)$, but is a zero for a certain branch $K$ of $H_{l}:=1+F_{l}(z) \cdot \overline{F_{l}}(\xi)$ or $Q_{j}:=1+G_{j}(z) \cdot \overline{G_{j}}(\xi)$. Consider the variety $Z_{p_{0}}$ defined by the coefficient polynomial of the degree zero term in the minimum polynomial of $K$. Write $X$ for the union of the branching varieties of $H_{l}, Q_{j}$. If $Z_{p_{0}} \not \subset X$, we move $p_{0}$ slightly such that $p_{0} \notin X$. Otherwise, we slightly move $p_{0}$ such that $p_{0}$ is a smooth point of $X$. Then we consider the Puiseux expansion of the involved branches of $G_{j}, H_{l}$ near $p_{0}$ and compare the vanishing orders near $p_{0}$. As in Lemma 2.2 (1), we finish the proof by applying (1.1).

We next formulate two elementary lemmas, which will be used in our induction argument later.

Lemma 2.3 Let $\left(a_{1}, \cdots, a_{p}\right)$ and $\left(b_{1}, \cdots, b_{p}\right)$ be $p$-tuples consisting of positive numbers with $p \geq 1$. Then there exists $i_{0}$ such that

$$
\frac{a_{i}}{a_{i_{0}}} \geq \frac{b_{i}}{b_{i_{0}}} \text { for all } i .
$$

Proof of Lemma 2.3: $i_{0}$ can be simply chosen so that $\frac{a_{i}}{b_{i}}$ achieves the minimum value when $i=i_{0}$.

Lemma 2.4 Let $F$ and $G$ be two vector valued holomorphic factions near $0 \in \mathbb{C}^{n}$. For any two non-negative integers $m_{1}, m_{1}$, there is a vector valued holomorphic function $H$ near $0 \in \mathbb{C}^{n}$ such that $\left(1+|F|^{2}\right)^{m_{1}} \cdot\left(1+|G|^{2}\right)^{m_{2}}=1+|H|^{2}$.

Proof of Lemma 2.4: When $m_{1}, m_{2}=0$, simply take $H=0$. The other case follows from the following elementary algebra formula: Let $a=\left(a_{1}, \cdots, a_{k}\right)$ and $b=\left(b_{1}, \cdots, b_{k}\right)$. Then

$$
\left(1+|a|^{2}\right)\left(1+|b|^{2}\right)=1+|a|^{2}+|b|^{2}+\sum_{j, l=1}^{k}\left|a_{j} b_{l}\right|^{2} .
$$




\section{Algebraicity for holomorphic maps satisfying transcen- dental functional equations}

In this section, we prove the algebraicity for germs of holomorphic functions satisfying a certain transcendental relation.

Proposition 3.1 Let $F_{l}: B \subset \mathbb{C}^{n} \rightarrow \mathbb{C}^{N_{l}}, G_{i}: B \subset \mathbb{C}^{n} \rightarrow \mathbb{C}^{N_{i}^{\prime}}$ be holomorphic maps defined over a small ball $B$ centered at the origin for $1 \leq l \leq m, 1 \leq i \leq v$ with $F_{l}(0), G_{j}(0)=0$. Let $\phi(z, \xi): B \times B \rightarrow \mathbb{C} \backslash\{0\}$ be a holomorphic algebraic function. Suppose that there exists $\mu_{l}, \lambda_{i} \in \mathbb{R}^{+}$such that

$$
\phi(z, \xi) \cdot \prod_{i=1}^{v}\left(1+G_{i}(z) \cdot \bar{G}_{l}(\xi)\right)^{\lambda_{i}}=\prod_{l=1}^{m}\left(1+F_{l}(z) \cdot \bar{F}_{l}(\xi)\right)^{\mu_{l}} \quad \text { over } B \times B
$$

Then there exist algebraic functions $\widehat{F}_{l}\left(z, X_{1}, \cdots, X_{v}\right)$ holomorphic for $\left(z, X_{1}, \cdots, X_{v}\right) \approx 0$ for all $1 \leq l \leq m$ such that $F_{l}(z)=\widehat{F}_{l}\left(z, G_{1}(z), \cdots, G_{v}(z)\right)$ for $z \approx 0$. In particular, when $v=0$, then each $F_{l}$ is algebraic. (Notice here, there is no restriction on $\mu_{j}$ except that they are positive.)

Proof of Proposition 3.1: Write $D_{\delta}=\frac{\partial}{\partial z_{\delta}}$ and for $\alpha=\left(\alpha_{1}, \cdots, \alpha_{n}\right) \in\left(\mathbb{Z}_{0}^{+}\right)^{n}$, write $D^{\alpha}=$

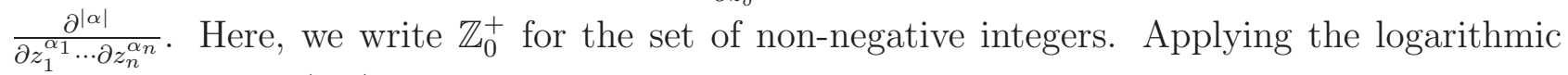
differentiation to (3.1), we get for any $1 \leq \delta \leq n$ and $z, \xi \approx 0$ the following:

$$
\sum_{l=1}^{m} \frac{\mu_{l} D_{\delta}\left(F_{l}\right)(z) \cdot \overline{F_{l}}(\xi)}{1+F_{l}(z) \cdot \overline{F_{l}}(\xi)}=\widehat{\phi}_{\delta}(z, \xi)+\sum_{i=1}^{v} \frac{\lambda_{i} D_{\delta}\left(G_{i}\right)(z) \cdot \overline{G_{i}}(\xi)}{1+G_{i}(z) \cdot \overline{G_{i}}(\xi)}
$$

where $\widehat{\phi}_{\delta}(z, \xi)=D_{\delta} \log \phi(z, \xi)$ is Nash algebraic in $(z, \xi)$ by the assumption. Write

$$
\chi=\left(\chi_{1}, \cdots, \chi_{N}\right)=\left(\sqrt{\mu_{1}} F_{1}, \cdots, \sqrt{\mu_{m}} F_{m}\right)
$$

with $N=N_{1}+\cdots N_{m}$. Then, we can rewrite (3.2) as follows:

$$
\sum_{l=1}^{N} D_{\delta}\left(\chi_{l}\right)(z) \cdot \bar{\chi}_{l}(\xi)+H_{\delta}(z, \bar{\chi}(\xi))=\Phi_{\delta}\left(z, \xi, \bar{G}_{1}(\xi), \cdots, \bar{G}_{v}(\xi)\right),
$$

where $\Phi_{\delta}\left(z, \xi, X_{1}(\xi), \cdots, X_{v}(\xi)\right)$ is a Nash algebraic function in $\xi$ and $X_{i}(\xi)$ for all $i$. Now, differentiating (3.3), we get for any $\alpha$ the following

$$
\sum_{l=1}^{N} D^{\alpha}\left(\chi_{l}\right)(z) \cdot \overline{\chi_{l}}(\xi)+H_{\alpha}(z, \bar{\chi}(\xi))=\Phi_{\alpha}\left(z, \xi, \bar{G}_{1}(\xi), \cdots, \bar{G}_{v}(\xi)\right) .
$$


Here for any $\alpha$ and for any fixed $z, H_{\alpha}(z, \bar{\chi}(\xi))$ is rational in $\bar{\chi}$, and has no constant and linear terms in the Taypor expansion with respect to $\bar{\chi}$. Also, $\Phi_{\alpha}\left(z, \xi, X_{1}, \cdots, X_{v}\right)$ is Nash algebraic in $\xi, X_{1}, \cdots, X_{v}$ for any fixed $z$.

We emphasize that, the advantage in the present situation is that $z, \xi$ are totally independent variables. Now, let $\mathcal{L}:=\operatorname{Span}_{\mathbb{C}}\left\{\left.D^{\alpha}(\chi(z))\right|_{z=0}\right\}|\alpha| \geq 1$ be a vector subspace of $\mathbb{C}^{N}$. Let $\left\{\left.D^{\alpha_{i}}(\chi(z))\right|_{z=0}\right\}_{i=1}^{\tau}$ be a basis for $\mathcal{L}$. Then for a small open ball $B_{0}$ centered at 0 in $\mathbb{C}^{n}, \chi\left(B_{0}\right) \subset \mathcal{L}$. Indeed, for any $z \approx 0$, we have from the Taylor expansion that $\chi(z)=$ $\chi(0)+\sum_{|\alpha| \geq 1} \frac{D^{\alpha}(\chi)(0)}{\alpha !} z^{\alpha}=\sum_{|\alpha| \geq 1} \frac{D^{\alpha}(\chi)(0)}{\alpha !} z^{\alpha} \in \mathcal{L}$.

Now, let $\nu_{i}(i=1 \cdots, N-\tau)$ be a basis of the Euclidean orthogonal complement of $\mathcal{L}$. Then, we have

$$
\nu_{i} \cdot \bar{\chi}(\xi)=0, \quad i=1, \cdots, N-\tau .
$$

Consider the system consisting of (3.4) at $z=0$ (with $\alpha=\alpha_{1}, \cdots, \alpha_{\tau}$ ) and (3.5). Since the Jacobian matrix of the functions in the left hand side of the system with respect to $\bar{\chi}$ at 0 is

$$
\left[\begin{array}{c}
\left.D^{\alpha_{1}}(\chi(z))\right|_{z=0} \\
\vdots \\
\nu_{N-\tau}
\end{array}\right]
$$

and is obviously invertible. Note that the left hand side of the system of equations consisting of (3.4) at $z=0$ (with $\alpha=\alpha_{1}, \cdots, \alpha_{\tau}$ ) and (3.5) is Nash algebraic in $\xi$ and the right hand side is Nash algebraic in $\xi, \bar{G}_{1}(\xi), \cdots, \bar{G}_{v}(\xi)$. By the algebraic version of the inverse function theorem (see, for instance [Hu1]), there exists Nash algebraic functions $\widehat{F}_{l}\left(\xi, X_{1}, \cdots, X_{v}\right)$ in all variables $\xi, X_{1}, \cdots, X_{v}$ for all $1 \leq l \leq m$ such that $F_{l}(\xi)=\frac{\chi_{l}}{\sqrt{\mu_{l}}}(\xi)=\widehat{F}_{l}\left(\xi, G_{1}(\xi), \cdots, G_{v}(\xi)\right)$ near $\xi=0$. This proves Proposition 3.1.

Remark 3.1 If one only has the following kind of equation

$$
\rho^{*}(F, \overline{F(z)})=k(z, \bar{z}) \rho(z, \bar{z})
$$

for a certain smooth function $k(z, \bar{z})$ with $\rho, \rho^{*}$ algebraic in their variables, then the differentiation can only be taken by $\mathrm{CR}$ vector fields tangent to and along the manifold defined by $\rho(z, \bar{z})=0$. The argument will be much more involved, and the algebraicity might be only achieved under certain non-degeneracy assumptions. This is the situation that one encounters in studying CR maps. (See [Hu1] for instance).

Let $F_{l}, G_{j}$ be holomorphic maps as in Proposition 3.1 satisfying the transcendental equation (3.1). It follows from Proposition 3.1 that there exists algebraic functions $\widehat{F}_{l}\left(z, X_{1}, \cdots, X_{v}\right)$ such that $F_{l}(z)=\widehat{F}_{l}\left(z, G_{1}(z), \cdots, G_{v}(z)\right)$ as germ of holomorphic functions at $z=0$ for all $l$. Let 
$N^{\prime}=N_{1}^{\prime}+\cdots N_{v}^{\prime}$ and let $g_{1}(z), \cdots, g_{N^{\prime}}(z)$ be all components in $G(z)=\left(G_{1}(z), \cdots, G_{v}(z)\right)$. Let $\mathfrak{R}$ be the field of rational functions in $z$ and consider the field extension

$$
\mathfrak{F}=\mathfrak{R}\left(g_{1}(z), \cdots, g_{N^{\prime}}(z)\right) .
$$

Let $K$ be the transcendence degree of the field extension $\mathfrak{F} / \mathfrak{R}$. If $K=0$, then each element in $\left\{g_{1}(z), \cdots, g_{N^{\prime}}(z)\right\}$ is a Nash algebraic function. Hence each $F_{l}(z)$ is also algebraic for all l. Otherwise, by re-ordering the lower index, let $\mathcal{G}=\left\{g_{1}, \cdots, g_{K}\right\}$ be the maximal algebraic independent subset in $g_{1}, \cdots, g_{N^{\prime}}$, and it follows that the transcendence degree of $\mathfrak{F} / \mathfrak{R}(\mathcal{G})$ is 0 . In fact, for any $l>K$, there exists a minimal polynomial $P_{l}\left(z, X_{1}, \cdots, X_{K}, X\right)$ such that $P_{l}\left(z, g_{1}(z), \cdots, g_{K}(z), g_{l}(z)\right) \equiv 0$ and moreover, $\frac{\partial P_{l}\left(z, X_{1}, \cdots, X_{K}, X\right)}{\partial X}\left(z, g_{1}(z), \cdots, g_{K}(z), g_{l}(z)\right) \not \equiv 0$ in $U$, a small neighborhood of 0 , for otherwise, $P_{l}$ can not be a minimal polynomial of $g_{l}$. Now the vanishing of the partial derivatives for all $l$ forms a proper local complex analytic variety near 0 . Let $\gamma:[0,1] \rightarrow U$ be a smooth simple curve with $\gamma(0)=0$ and $\gamma((0,1])$ does not curve the just mention variety. Applying the algebraic version of the existence and uniqueness part of the implicit function theorem, there exist a small connected open subset $U_{0} \subset U$ with $0 \in \overline{U_{0}}$ and a holomorphic algebraic function $\widehat{g}_{l}$ in the neighborhood $\widehat{U_{0}}$ of $\left\{\left(z, g_{1}(z), \cdots, g_{K}(z)\right): z \in U_{0}\right\}$ in $\mathbb{C}^{n} \times \mathbb{C}^{K}$, such that $g_{l}(z)=\widehat{g}_{l}\left(z, g_{1}(z), \cdots, g_{K}(z)\right)$ for any $z \in U_{0}$. (We can assume that $U_{0}$ is the projection of $\widehat{U_{0}}$.) Substitute into $\widehat{F}_{l}\left(z, G_{1}(z), \cdots, G_{v}(z)\right)$, and still denote it, for simplicity of notation, by $\widehat{F}_{l}\left(z, g_{1}(z), \cdots, g_{K}(z)\right)$ with

$$
\widehat{F}_{l}\left(z, g_{1}(z), \cdots, g_{K}(z)\right)=\widehat{F}_{l}\left(z, G_{1}(z), \cdots, G_{v}(z)\right) \text { for } z \in U_{0} .
$$

Write $\widehat{G}_{j}\left(z, g_{1}(z), \cdots, g_{K}(z)\right)$ for $\widehat{g}$ when $j>K$ and $\widehat{G_{j}}\left(z, g_{1}(z), \cdots, g_{K}(z)\right)=g_{j}$ for $j \leq K$. Now, let $X=\left(X_{1}, \cdots, X_{K}\right)$ and replace $g_{i}(\xi)$ by $X_{i}$ for $1 \leq i \leq K$ in $F_{l}(\xi)=\widehat{F}_{l}\left(\xi, g_{1}(\xi), \cdots, g_{K}(\xi)\right)$ and $G_{j}(\xi)=\widehat{G}_{j}\left(\xi, g_{1}(\xi), \cdots, g_{K}(\xi)\right)$, in the following quantity:

$$
\sum_{l=1}^{m} \frac{\mu_{l} \overline{D_{z_{\delta}}\left(F_{l}\right)(z)} \cdot F_{l}(\xi)}{1+\overline{F_{l}(z)} \cdot F_{l}(\xi)}-\sum_{j=1}^{v} \frac{\lambda_{i} \overline{D_{z_{\delta}}\left(G_{j}\right)(z)} \cdot G_{j}(\xi)}{1+\overline{G_{j}(z)} \cdot G_{j}(\xi)}-\frac{D_{\bar{z}_{\delta}} \bar{\phi}(\bar{z}, \xi)}{\bar{\phi}(\bar{z}, \xi)}
$$

Denote the new quantity by $\Phi_{\delta}(\bar{z}, \xi, X)$. We have the following:

Lemma $3.1 \Phi_{\delta}(\bar{z}, \xi, X) \equiv 0$ for any $1 \leq \delta \leq n$, for any $z$ near 0 and any $(\xi, X) \in \widehat{U_{0}}$.

Proof of Lemma 3.1: Suppose not. Notice that $\Phi_{\delta}(\bar{z}, \xi, X)$ is Nash algebraic in $(\xi, X)$ by Proposition 3.1. For a generic fixed $z=z_{0}$ near 0 , since $\Phi_{\delta}\left(\bar{z}_{0}, \xi, X\right) \not \equiv 0$, there exist polynomials $A_{\alpha}(\xi, X)$ for $0 \leq \alpha \leq m$ with $A_{0}(\xi, X) \not \equiv 0$ such that

$$
\sum_{0 \leq \alpha \leq m} A_{\alpha}(\xi, X) \Phi_{\delta}^{\alpha}\left(\bar{z}_{0}, \xi, X\right) \equiv 0 .
$$

As $\Phi_{\delta}\left(\overline{z_{0}}, \xi, g_{1}(\xi), \cdots, g_{K}(\xi)\right) \equiv 0$ for $\xi \in U_{0}$, then it follows that $A_{0}\left(\xi, g_{1}(\xi), \cdots, g_{K}(\xi)\right) \equiv 0$ for $\xi \in U_{0}$. This is a contradiction to the assumption that $\left\{g_{1}(\xi), \cdots, g_{K}(\xi)\right\}$ is an algebraic independent set.

We now are in a position to prove the following algebraicity result: 
Theorem 3.1 Let $\left(\lambda_{1}, \cdots, \lambda_{v}\right)$ and $\left(\mu_{1}, \cdots, \mu_{m}\right)$ be two sets of positive real numbers satisfying the following condition:

$$
\operatorname{span}_{\mathbb{Q}_{0}^{+}}\left\{\lambda_{j}\right\}_{j=1}^{v} \cap \operatorname{span}_{\mathbb{Q}_{0}^{+}}\left\{\mu_{l}\right\}_{l=1}^{m}=\{0\} .
$$

Let $B$ be a small ball centered at the origin of $\mathbb{C}^{n}$. Let $F_{l}: B \rightarrow \mathbb{C}^{N_{l}}, G_{j}: B \rightarrow \mathbb{C}^{N_{j}^{\prime}}$ be non-constant holomorphic maps with $F_{l}(0), G_{j}(0)=0$ satisfying the transcendental equation:

$$
\prod_{l=1}^{m}\left(1+F_{l}(z) \cdot \bar{F}_{l}(\xi)\right)^{\mu_{l}} \cdot \prod_{i=1}^{v}\left(1+G_{i}(z) \cdot \bar{G}_{l}(\xi)\right)^{-\lambda_{i}}=\phi(z, \xi) .
$$

Suppose that $\phi(z, \xi)$ is holomorphic and algebraic over $B \times B$. Then $F_{l}$ and $G_{j}$ are Nash algebraic.

Proof of Theorem 3.1: Keep the same notation we set up above. After a unitary transformation to simplify $F_{l}, G_{j}$, if needed, we may assume in what follows that the component functions in $F_{l}$ or $G_{j}$ are linearly independent over $\mathbb{C}$. Write

$$
\begin{gathered}
\Psi(z, \xi, X)= \\
=\log \left(\prod_{l=1}^{m}\left(1+\overline{F_{l}(z)} \cdot \widehat{F}_{l}(\xi, X)\right)^{\mu_{l}}\right)-\log \left(\prod_{j=1}^{v}\left(1+\overline{G_{j}(z)} \cdot \widehat{G}_{j}(\xi, X)\right)^{\lambda_{j}}\right)-\log \bar{\phi}(\bar{z}, \xi) .
\end{gathered}
$$

Lemma 3.1 shows that for any $z$ near 0 , and $(\xi, X) \in \widehat{U_{0}}$ as defined there, $\frac{\partial}{\partial \bar{z}_{\delta}} \Psi(z, \xi, X)=0$. Notice that $\frac{\partial}{\partial z_{\delta}} \Psi(z, \xi, X)=0$ as $\Psi(z, \xi, X)$ is anti-holomorphic in $z$. Since $\Psi(0, \xi, X)=0$, then $\Psi(z, \xi, X) \equiv 0$ for $z \in B$ and $(\xi, X) \in \widehat{U_{0}}$. Hence, we arrive at the following identity,

$$
\prod_{l=1}^{m}\left(1+\overline{F_{l}(z)} \cdot \widehat{F}_{l}(\xi, X)\right)^{\mu_{l}}=\bar{\phi}(\bar{z}, \xi) \prod_{j=1}^{v}\left(1+\overline{G_{j}(z)} \cdot \widehat{G}_{j}(\xi, X)\right)^{\lambda_{j}} \quad(z, \xi, X) \in B \times \widehat{U_{0}}
$$

Here $\widehat{F}_{l}$ and $\widehat{G}_{j}$ are algebraic in their variables. Notice that when $\widehat{F}_{l}$ and $\widehat{G}_{j}$ are independent of $X$, then $F_{l}$ and $G_{j}$ are already algebraic. Hence, we will assume, in the course of the proof of the theorem, that one of the maps from $\left\{\widehat{F}_{l}\right\}$ or $\left\{\widehat{G}_{j}\right\}$ depends on $X$. Let $h(\xi, X)$ be one of these. Let $E$ be the zero defined by the prime factors involving only $\xi$ in the non-zero polynomial coefficients of a minimal polynomial of $h(\xi, X)$. Then for a fixed $\xi \notin B \backslash E, h(\xi, X)$ is not constant in $X$. Now, choose $X_{\xi}$ such that $\left(\xi, X_{\xi}\right) \in \widehat{U_{0}}$. Then for any unit vector $v$ in $\mathbb{C}^{K}, h(\xi, t):=h\left(\xi, X_{\xi}+t v\right)$ is algebraic and holomorphic in $t(\approx 0) \in \mathbb{C}$. For a generic choice of the unit vector $v, h(\xi, t)$ is a non-constant algebraic function in $t$. Also fix such a vector $v$. Now holomorphically continuing $h(\xi, t)$ along loops in $\mathbb{C}$ avoiding its branch points $\mathcal{B}$, we get multiple valued functions: $\left\{h_{1}(\xi, t), \cdots, h_{m}(\xi, t)\right\}$. If for any compact subset $L,\left|h_{j}(\xi, t)\right| \leq C_{L}$ for $t \in L \backslash \mathcal{B}$ with $C_{L}$ a constant depending only on $L$, then any symmetric function of them is 
holomorphic over $L$. Certainly not all symmetric functions of them can be uniformly bounded in $\mathbb{C}$, for, otherwise, by the Louville theorem, all symmetric functions of $\left\{h_{j}(\xi, t)\right\}$ are constant and thus each $h_{j}$ is constant in $t$, which contradicts to the assumption. Hence, in this setting, we must have an unbounded branch near $t=\infty$. Let $t_{0} \in \mathbb{C}$ be such that some branches of $h$ are unbounded near $t_{0}$. For simplicity of notation, assume $t_{0}=\infty$. Let $\gamma:[0,1] \rightarrow \mathbb{C}$ be a curve connecting 0 to a point close to $t_{0}=\infty$ and continue $h$ along $\gamma$ to get a branch, which is still denoted by $h$, near $\gamma(1)$. By what we argued above, we can assume that the continuation of such an $h$ along curves near $\infty$ leads to unbounded multi-valued functions near $t=\infty$.

For simplicity of notation, assume that the $h\left(\xi, X_{\xi}+t v\right)$ mentioned above is $\widehat{F}_{1}\left(\xi, X_{\xi}+t v\right)$. We can also assume that $|\gamma(1)|>R_{0}$ and all branching locus (other that $\infty$ ) of $F_{l}(\xi, t):=$ $\widehat{F}_{l}\left(\xi, t v+X_{\xi}\right), G_{j}(\xi, t):=\widehat{G}_{j}\left(\xi, t v+X_{\xi}\right)$ are inside the disk $|t|<R_{0}$. Also, we can assume that $\gamma$ does not hit the branching locus of $F_{l}(\xi, t)$ and $G_{j}(\xi, t)$.

Write the Puiseux expansion of $h$ at $t=\infty$ as follows:

$$
h(\xi, t)=a_{\xi i_{0}} t^{\frac{i_{0}}{N_{\xi}}}(1+o(1)),
$$

where $N_{\xi}, i_{0} \in \mathbb{N}, a_{\xi i_{0}} \neq 0$. We will fix $\xi \in U_{0} \backslash E$ and $v$ as above for the rest of the proof. Restricting (3.9) to $X=t v+X_{\xi}$ and denoting $\widehat{F}_{l}\left(\xi, t v+X_{\xi}\right), \widehat{G}_{j}\left(\xi, t v+X_{\xi}\right)$ by $\widehat{F}_{l}(\xi, t), \widehat{G}_{j}(\xi, t)$ respectively for all $l, j$ as before, one has the following equation for $t \approx 0$ :

$$
\prod_{l=1}^{m}\left(1+\overline{F_{l}(z)} \cdot \widehat{F}_{l}(\xi, t)\right)^{\mu_{l}}=\bar{\phi}(\bar{z}, \xi) \prod_{j=1}^{v}\left(1+\overline{G_{j}(z)} \cdot \widehat{G}_{j}(\xi, t)\right)^{\lambda_{j}} .
$$

If either a certain $\widehat{F}_{l}(\xi, t)$ for some $l \neq 1$ or a certain $\widehat{G}_{j}(\xi, t)$ for some $j$, obtained by continuing along $\gamma$ to $\gamma(1)$, has bounded branches by continuing in the annulus $R_{0}<|t|<\infty$, then there is an $\epsilon_{0}$ such that for $|z|<\epsilon_{0}$, one has the following for $|t|>R_{0}$ :

$$
\frac{1}{2}<\left|1+\overline{F_{l}(z)} \cdot \widehat{F}_{l}(\xi, t)\right| \leq 2 \text { or } \frac{1}{2}<\left|1+\overline{G_{j}(z)} \cdot \widehat{G}_{j}(\xi, t)\right| \leq 2 .
$$

Otherwise,

$$
1+\overline{F_{l}(z)} \cdot \widehat{F}_{l}(\xi, t)=\sum_{\left\{i \mid s_{i}=s_{l}\right\}} \overline{f_{l i}(z)} a_{\xi l s_{i}} t^{\frac{s_{i}}{N_{\xi}}}+o\left(t^{\frac{s_{l}}{N_{\xi}}}\right) \text { for } t \text { near } \infty
$$

and for $s_{i} \in \mathbb{Z}_{0}^{+}, s_{l}=\max _{i} s_{i}>0$, where $F_{l}(z)=\left(\cdots, f_{l i}(z), \cdots\right), s_{i} / N_{\xi}$ is the order of blowing up of $f_{l i}$. In addition, $\sum_{\left\{i \mid s_{i}=s_{l}\right\}} \overline{f_{l i}(z)} a_{\xi l s_{i}} \not \equiv 0$ by the arrangement that components in $F_{l}(z)$ are not linearly dependent. We have a similar analysis for $1+\overline{G_{j}(z)} \cdot \widehat{G}_{j}(\xi, t)$. Hence, for unbounded branches, we have at $t=\infty$ :

$$
1+\overline{F_{l}(z)} \cdot \widehat{F}_{l}(\xi, t)=\overline{a_{\xi l}(z)} t^{\frac{s_{l}}{N_{\xi}}}+o\left(t^{\frac{s_{l}}{N_{\xi}}}\right), \quad 1+\overline{G_{j}(z)} \cdot \widehat{G}_{j}(\xi, t)=\overline{b_{\xi j}(z)} t^{\frac{t_{j}}{N_{\xi}}}+o\left(t^{\frac{t_{j}}{N_{\xi}}}\right)
$$


for $a_{\xi l}(z), b_{\xi j}(z) \not \equiv 0$ and $N_{\xi}, s_{l}, t_{j} \in \mathbb{N}$. Notice $\widehat{F}_{1}$ is one of them with $s_{1}>0$. Now, we let $\left|z_{0}\right|<\epsilon_{0}$ be such that $a_{\xi l}\left(z_{0}\right), b_{\xi j}\left(z_{0}\right) \neq 0$. Fix such a $z_{0}$. We perturb slightly $\gamma$ also to make sure that no terms in both sides of (3.11) hit zero. Moreover, the perturbed $\gamma$ has the same terminal points and, relative to the terminal points, it has the same homopotic class as the previous one in the space:

$\mathbb{C} \backslash\left\{\right.$ branching points of $\widehat{F}_{l}(\xi, t), \widehat{G}_{j}(\xi, t)$ and zeros of $1+\overline{F_{l}\left(z_{0}\right)} \cdot \widehat{F}_{l}(\xi, t), 1+\overline{G_{j}\left(z_{0}\right)} \cdot \widehat{G}_{j}(\xi, t)$ in $\left.t\right\}$.

By holomorphic continuation of (3.11) with $z=z_{0}$ along the curve $\gamma(\tau)$ in $\tau$ from $\tau=0$ to $\tau=1$, the equation (3.11) holds for certain fixed branches of $\widehat{F}_{l}(\xi, t)$ and $\widehat{G}_{j}(\xi, t)$ with $z=z_{0}$ and $t$ near $p_{0}=\gamma(1)$

Hence, it follows from equation (3.11) with $z=z_{0}, t \approx p_{0}$, the Puiseux expansions in (3.12) centered at $t=\infty$, and the blowing up rate at $t=\infty$ that

$$
\prod_{S_{l}>0}\left(\overline{a_{\xi l}\left(z_{0}\right)} t^{\frac{s_{l}}{N_{\xi}}}\right)^{\mu_{l}}=c\left(z_{0}, \xi\right) \bar{\phi}\left(\bar{z}_{0}, \xi\right) \prod_{t_{j}>0}\left(\overline{b_{\xi j}\left(z_{0}\right)} t^{\frac{t_{j}}{N_{\xi}}}\right)^{v_{j}} .
$$

Here $s_{1}>0$ and $s_{l}=0, t_{j}=0$ for those bounded branches. Also $c\left(z_{0}, \xi\right)$ has the property that

$$
\left|c\left(z_{0}, \xi\right)\right|, \frac{1}{\left|c\left(z_{0}, \xi\right)\right|}<\infty
$$

Comparing the blowing-up rate for $t$ near $\infty$, one has:

$$
\sum_{s_{l}>0} \mu_{l} s_{l}=\sum_{t_{j}>0} v_{j} t_{j}
$$

This contradicts the assumption in (1.1).

We thus proved that all $\widehat{F}_{l}(\xi, X)$ and $\widehat{G}_{j}(\xi, X)$ can not depend on $X$. Hence $F_{l}(\xi), G_{j}(\xi)$ are algebraic.

\section{Single-valuedness of algebraic functions satisfying tran- scendental functional equations}

In this section, we derive the single-valuedness for algebraic functions satisfying the transandental functional equation (3.1).

\subsection{Starting point of the induction}

We first start with the following lemma: 
Lemma 4.1 Let $F_{l}: B \rightarrow \mathbb{C}^{N_{l}}$ be algebraic and holomorphic for $1 \leq l \leq m$ with $B$ a ball centered at the origin. Let $h(z, \xi)$ be a rational and holomorphic function in $B$ such that $h(z, \bar{z})>0$ for $z \in B$. Suppose that

$$
\prod_{l=1}^{m}\left(1+\left|F_{l}(z)\right|^{2}\right)^{\mu_{l}}=h(z, \bar{z}) \text { on } B,
$$

for $\mu_{l} \in \mathbb{R} \backslash\{0\}$. Then $F_{l}$ is a rational function for each $1 \leq l \leq m$.

Proof of Lemma 4.1: There is a proper complex analytic variety $E$ such that any branch of $F_{l}$ extends holomorphically along any path $\gamma \subset \mathbb{C}^{n} \backslash E$. Moreover, for any $q \in \mathbb{C}^{n} \backslash E$, the total number $\nu(q)$ of all possible (different) values obtained by continuing $F$ along closed curves in $\mathbb{C}^{n} \backslash E$ starting from a certain branch of $F$ near $q$ and then coming back and evaluating at $q$, is independent of $q \in \mathbb{C}^{n} \backslash E$. When $\nu(q)=1, F$ is single-valued. Assume that $q \in B \backslash E$ is sufficiently close to 0 . Write $U_{l}$ for an $\left(N_{l}+1\right) \times\left(N_{l}+1\right)$ unitary matrix such that

$$
\widetilde{F}_{l}=\left(\widetilde{F}_{l, 0}, \cdots, \widetilde{F}_{l, N_{l}}\right)=\left(1, F_{l}\right) \cdot U_{l}
$$

has the property that $\widetilde{F}_{l}(q)=\left(c_{l}, 0, \cdots, 0\right)$. Then $|\widetilde{F}|^{2}=1+\left|F_{l}\right|^{2}$. Write

$$
G_{l}=\left(G_{l, 1}, \cdots, G_{l, N_{l}}\right):=\left(\frac{\widetilde{F}_{l, 2}}{\widetilde{F}_{l, 1}}, \cdots, \frac{\widetilde{F}_{l, N_{l}}}{\widetilde{F}_{l, 1}}\right)
$$

Write $h(z, \bar{z})=h(q, \bar{q})+2 \Re h_{1}(z)+h_{m i x}(z, \bar{z})$ with $h_{1}(z)=h(z, \bar{q})-h(q, \bar{q})$. Then the rational function $h_{1}$ takes the zero value at $q$ and holomorphic in a neighborhood of $q$. Moreover, the real-valued rational function $h_{m i x}(z, \bar{z})$ only has mixed terms in its Taylor expansion at $(q, \bar{q})$. Notice that $h(q, \bar{q})>0$ and $h_{\text {mix }}(q, \bar{q})=0$. Taking $\partial \bar{\partial} \log$ to (4.1) and noticing that $|\widetilde{F}|^{2}=1+\left|F_{l}\right|^{2}$, we get

$$
\sum_{l=1}^{m} \mu_{l} \partial \bar{\partial} \log \left(1+\left|G_{l}(z)\right|^{2}\right)=\partial \bar{\partial} \log h(z, \bar{z})
$$

Write

$$
h(z, \bar{z})=h(q, \bar{q})\left(1+\frac{h_{1}(z)}{h(q, \bar{q})}\right)\left(1+\frac{\overline{h_{1}(z)}}{h(q, \bar{q})}\right) \widetilde{h}(z, \bar{z}) .
$$

Then $\widetilde{h}(z, \bar{z})-1$ has only mixed terms in its Taylor expansion at $(q, \bar{q})$. Since $\partial \bar{\partial} \log [(1+$ $\left.\left.\frac{h_{1}(z)}{h(q, \bar{q})}\right)\left(1+\frac{\overline{h_{1}(z)}}{h(q, \bar{q})}\right)\right]=0$, comparing the Taylor expansion at $(q, \bar{q})$ for the left and right hand sides of (4.2), after taking away $\partial \bar{\partial}$, we get

$$
\prod_{l=1}^{m}\left(1+\left|G_{l}(z)\right|^{2}\right)^{\mu_{l}}=\widetilde{h}(z, \bar{z}) .
$$


Notice that $\widetilde{h}(q, \bar{q})=1$. Now for any closed (simple) curve $\gamma \in \mathbb{C}^{n} \backslash E$ with $\gamma(0)=\gamma(1)=q$, after perturbing $\gamma$ slightly in the part $\gamma[\delta, 1-\delta]$ for a small $\delta$ to avoid the zeros of $F_{l, 0}, G_{l}(z)$ extends holomorphically along $\gamma$. From the right hand side of $(4.3)$, we see that $\widetilde{h}(z, \bar{z})$ is real analytic along $\gamma$, too. By the uniqueness of real analytic functions, we see that (4.2) holds along $\gamma$. Since $\widetilde{h}$ is single-valued and $\widetilde{h}(q, \bar{q})=1$, we see that the value of $G_{l}$ continued along $\gamma$ still takes 0 -value at $q$ for each $l$. (This is the only way to make sure the right hand side of (4.3) attains its minimum value 1.) This shows that $G_{l}$ is single-valued for each $l$. Now, notice that $\widetilde{F}_{l} \cdot U_{l}^{-1}=\left(1, F_{l}\right)$. We have a certain non-zero linear combination of $\widetilde{F}_{l}$ which is 1 . Dividing on both sides of such a linear combinantion by $F_{l, 0}$, we see that $F_{l, 0}$ and thus $F_{l}$ are all single valued. Hence $F_{l}$ are rational.

Remark 4.2 There is an elegant argument by Mok in [M3] for dealing with the single-valuedness of multi-valued maps, based on the Cauchy-Schwarz inequality, which can also be used in our lemma here. Our argument for proving the single-valuedness of $F$ is based on the fact that $1+|\cdot|^{2}$ has the only extremal value at 0 and the group $S U(n, 1)$ acts transitively on the quadratic form: $|z|^{2}=|t|^{2}$. This argument applies to other type of potential functions which may not have the Schwarz inequality property but have similar minimum or maximum properties and symmetry. For instance, it applies similarly to the following functional equation:

$$
\prod_{l=1}^{m}\left(1-\left|F_{l}(z)\right|^{2}\right)^{\mu_{l}}=\chi(z, \bar{z}), \mu_{l}>0, \quad|z|<<1 .
$$

Here $F_{l}^{\prime} s$ are holomorphic in a small neighborhood of 0 with $F_{l}(0)=0$, and extend along curves without hitting a certain proper complex analytic variety, $\chi(z, \bar{z})>0$ is real analytic in $|z|<1$ and $\chi(z, \xi)$ is meromorphic in $\{|z|<1\} \times\{|\xi|<1\}$. Then one can also conclude the single-valuedness of each $F_{l}$ in the unit ball.

Corollary 4.1 Let $F_{l}: B \rightarrow \mathbb{C}^{N_{l}}$ be a non-constant algebraic and holomorphic map for each $1 \leq l \leq m$ with a ball $B$ centered at the origin. Suppose that there exists a non-constant holomorphic irreducible polynomial function $h(z, \xi)$ over $B \times B$ with $h(z, \bar{z})>0$ for $z \in \mathbb{C}^{n}$ such that

$$
\prod_{l=1}^{m}\left(1+F_{l}(z) \cdot \overline{F_{l}(z)}\right)^{\mu_{l}}=h(z, \bar{z})^{r} \quad \text { for } z \in B,
$$

and $\mu_{l} \in \mathbb{R}^{+}, r \in \mathbb{R}$. There are a positive integer $m_{l}$ and a positive constant $A_{l}$ such that $1+F_{l}(z) \cdot \overline{F_{l}(z)}=A_{l} h^{m_{l}}(z, \bar{z})$ for each $l$.

Proof of Corollary 4.1: After taking an $r^{\text {th }}$-root in (4.5), it follows from Lemma 4.1 that each $F_{l}$ is a rational function, for $1 \leq l \leq m$. Since $h$ is a polynomial, $F_{l}$ is bounded on any compact subset of $\mathbb{C}^{n}, F_{l}$ thus is a polynomial. Since the zeros of $\left(1+F_{l}(z) \bar{F}_{l}(\xi)\right)$ can only take along the zero set defined by $h(z, \xi)=0$, we finish the proof. 


\subsection{Proof of Theorem 4.1}

We now are in a position to prove the main result of this section:

Theorem 4.1 Let $h(z, \xi)$ be an irreducible polynomial function over $\mathbb{C}^{n} \times \mathbb{C}^{n}$ for $n \geq 1$ such that $h(z, \bar{z})>0$. Let $F_{l}(z): B \subset \mathbb{C}^{n} \rightarrow \mathbb{C}^{N_{l}}, G_{j}(z): B \subset \mathbb{C}^{n} \rightarrow \mathbb{C}^{N_{j}^{\prime}}$ be nonconstant holomorphic and algebraic over a small ball in $\mathbb{C}^{n}$ centered at 0 for $1 \leq l \leq m, 1 \leq j \leq v$. Suppose the following transcendental functional identity holds:

$$
\begin{aligned}
h(z, \bar{z})^{r} & =\prod_{l=1}^{m}\left(1+\left|F_{l}(z)\right|^{2}\right)^{\mu_{l}} \cdot \prod_{j=1}^{v}\left(1+\left|G_{j}(z)\right|^{2}\right)^{-\lambda_{j}} \quad \text { over } B, \text { or equivalently, } \\
h(z, \xi)^{r} & =\prod_{l=1}^{m}\left(1+F_{l}(z) \cdot \bar{F}_{l}(\xi)\right)^{\mu_{l}} \cdot \prod_{j=1}^{v}\left(1+G_{j}(z) \cdot \bar{G}_{j}(\xi)\right)^{-\lambda_{j}} \quad \text { over } B \times B,
\end{aligned}
$$

for $\mu_{l}, \lambda_{j} \in \mathbb{R}^{+}$satisfying (1.1), and for $r \in \mathbb{R} \backslash\{0\}$. Then $F_{l}, G_{j}$ are holomorphic polynomials for all $l, j$. In fact, there exist $m_{l}, n_{j} \in \mathbb{N}$, such that

$$
1+F_{l}(z) \cdot \bar{F}_{l}(\xi)=A_{l} h(z, \xi)^{m_{l}} \text { and } 1+G_{j}(z) \cdot \bar{G}_{j}(\xi)=B_{j} h(z, \xi)^{n_{j}},
$$

for certain $A_{l}, B_{j} \in \mathbb{R}^{+}$. Moreover,

$$
r=\sum_{l=1}^{m} m_{l} \mu_{l}-\sum_{j=1}^{v} \lambda_{j} n_{j}
$$

Write $H_{l}(z, \xi)=1+F_{l}(z) \cdot \bar{F}_{l}(\xi)$ and $Q_{j}(z, \xi)=1+G_{j}(z) \cdot \bar{G}_{j}(\xi)$. Then $H_{l}(z, \xi), Q_{j}(z, \xi)$ are holomorphic and algebraic over $B \times B$. Complexifying (4.6), we get, after possibly shrinking $B$ a little, the following:

$$
h(z, \xi)^{r}=\prod_{l=1}^{m} H_{l}^{\mu_{l}}(z, \xi) \cdot \prod_{j=1}^{v} Q_{j}^{-\lambda_{j}}(z, \xi) \text { over } B \times B .
$$

Now, let $X_{z}$ be the union of branching varieties of $F_{l}(z), G_{j}(z)$ and let $X$ be the union of branching varieties of $H_{l}(z, \xi)$ and $G_{j}(z, \xi)$ for $l=1, \cdots, m, j=1, \cdots, v$. Then $F$ and $G$ can be continued holomorphically along any path in $\mathbb{C}^{n} \backslash X_{z}$.

Our goal is to show that both $F$ and $G$ are forced to be single-valued from the identity (4.8). Notice that the argument in the proof of Corollary 4.1 can not be applied here, for now the potential functions involved do not have any required extremal property. Indeed, without the assumption in (1.1), one can easily see that $F, G$ do not have to be rational. 
Remark 4.3 We mention that there are many non-single-valued algebraic functions which only have zeros or points of indeterminancy along the zero set of an irreducible polynomial, say, $1+z \cdot \xi$. Indeed, let $Y_{1}$ and $Y_{2}$ be the solutions of the polynomial $Y^{2}+g(z) Y+(1+z \cdot \xi)$ with $g(0)=0$. Then $Y_{1}, Y_{2}$ are holomorphic near 0 and $Y_{1} \cdot Y_{2}=1+z \cdot \xi$. Hence, $Y_{1}, Y_{2}$ can only have zeros in $1+z \cdot \xi=0$. Also, we can find algebraic functions that have no zero at all, by simply considering the solutions of polynomial equation $Y^{2}+(z \cdot \xi) Y+1=0$. Hence, just by studying the distribution of zeros or points of indeterminancy, we can not conclude the single-valuedness of multi-valued maps.

The proof of Theorem 4.1 is done by a long and tedious induction argument based essentially on the monodromy analysis. We will show that (1.1) will be violated if some $F_{l}$ or $G_{j}$ fails to be single-valued.

Proof of Theorem 4.1: We first notice that under the assumption of Theorem 4.1, all components from $F_{l}$ and $G_{j}$ have leading coefficient 1 in their minimal polynomials. We next fix more notations. When there are $\alpha-H$ 's and $\beta$-Q's, we say that we are in the situation with $(\alpha, \beta)$-factors. We use $r_{*}, r_{* *}$ to represent rational numbers, $n_{* *}, m_{* *}, k_{* * *}$ to denote integers, $n_{* *}^{+}, m_{* *}^{+}, k_{* * *}^{+}$to denote positive integers and $\iota_{*}, A$ to denote real numbers. All of them may be different in different contexts.

Step 0: $H_{l}^{N_{0}}$ or $Q_{j}^{N_{0}}$ is a rational function. Assume $H_{l}(z, \xi)$ (resp. $\left.Q_{j}(z, \xi)\right)$ is a rational function for some $l$ (resp. for some $j$ ). As all branches of $H_{l}$ (resp. $G_{j}$ ) remain uniformly bounded over any compact subset, then $H_{l}$ (resp. $G_{j}$ ) must be a polynomial. By Lemma 2.2, the zero of $H_{l}(z, \xi)$ ( resp. $\left.Q_{j}(z, \xi)\right)$ is contained in the variety defined by $\{(z, \xi): h(z, \xi)=0\}$ (resp. $\{(z, \xi): h(z, \xi)=0\})$. Hence, we conclude that $H_{l}(z, \xi)=A_{l} h(z, \xi)^{m_{l}^{+}}$(resp. $Q_{j}(z, \xi)=$ $\left.B_{j} h(z, \xi)^{n_{j}^{+}}\right)$.

If for some $l$, all branching varieties of $H_{l}(z, \xi)$ are simple cyclic branching varieties with respect to any branch, then there exists an $N_{0} \in \mathbb{N}$ such that $H_{l}^{N_{0}}$ is a polynomial. Again, we get $H_{l}(z, \xi)^{N_{0}}=A_{l}^{\prime} h(z, \xi)^{m_{l}^{+}}$. Hence, $F_{l}$ is a polynomial by Corollary 4.1. Now, if for each $l$, $H_{l}$ is single-valued, applying Corollary 4.1 again, we also conclude that $Q_{j}^{\prime} s$ and thus $G_{j}^{\prime} s$ are all single-valued polynomails. Hence the theorem is done in this setting. Similar arguments apply when for each $\left.j, Q_{j}(z, \xi)\right)$ has only simple cyclic branching varieties with respect to any branch. We then apply Lemma 4.1 to $H_{l}$ (resp. $Q_{j}$ ) to be single-valued.

From now on, we always assume that $m, v \geq 1$, and also assume that some $H_{l}$ and some $Q_{j}$ have non-cyclic branching varieties. We will prove Theorem 4.1 by an induction argument on $m$ and $v$. When $m=0$, we are done by Corollary 4.1. We first consider the case of $(m, v)$-factor with $m=1, v>0$ to illustrate our general argument.

Step 1: $(1, v)$-factor. We assume that Theorem 4.1 is proved for the $(1, j)$-factor case with $0 \leq j \leq \beta-1$ and we consider the case with $(1, \beta)$-factors. The following identity is the basic assumption:

$$
h^{r}=H_{1}^{\mu_{1}} Q_{1}^{-\lambda_{1}} \cdots Q_{\beta}^{-\lambda_{\beta}} \text { near } \quad 0 .
$$


Step 1.1: As before, we can assume $H_{1}$ has an irreducible non-cyclic branching hypervariety $E$ with respect to a certain branch of $H_{1}$. Applying Lemma 2.1 to (4.9), we have

$$
m_{11}^{+} \mu_{1}=n_{11} \lambda_{1}+\cdots+n_{1 \beta} \lambda_{\beta}
$$

and

$$
h^{m_{11}^{+} r}=\left(H_{1}^{-n_{11}} Q_{1}^{m_{11}^{+}}\right)^{-\lambda_{1}} \cdots\left(H_{1}^{-n_{1 v}} Q_{\beta}^{m_{11}^{+}}\right)^{-\lambda_{\beta}} .
$$

Case 1.1.1: If $\left(H_{1}^{-n_{11}} Q_{1}^{m_{11}^{+}}\right)^{N_{0}}$ is rational for some $N_{0} \in \mathbb{N}$, then we have

$$
H_{1}^{-n_{11}} Q_{1}^{m_{11}^{+}}=A h(z, \xi)^{r_{1}}
$$

for $r_{1} \in \mathbb{Q}$, since the zeros and points of indeterminancy of $\left(H_{1}^{-n_{11}} Q_{1}^{m_{11}^{+}}\right)^{N_{0}}$ have to be along $E_{0}=\{(z, \xi): h(z, \xi)=0\}$.

Now, if $n_{11}<0$, by Corollary 4.1 with $(2,0)$-factors, we conclude that $Q_{1}=B_{1} h(z, \xi)^{r_{2}}$ with $r_{2} \in \mathbb{N}$. Hence, after a cancelation, we reduce (4.9) to the $(1, \beta-1)$-factor case. Thus we are done by induction.

If $n_{11}>0$, then we get by (4.12):

$$
H_{1}=A Q_{1}^{\frac{m_{11}^{+}}{n_{11}}} h(z, \xi)^{r_{2}} \text { or } Q_{1}=A H_{1}^{\frac{n_{11}}{m_{11}^{+}}} h(z, \xi)^{r_{3}} .
$$

Substituting into (4.9), we get

$$
Q_{1}^{-\left(\lambda_{1}-\frac{m_{11}^{+}}{n_{11}} \mu_{1}\right)} Q_{2}^{-\lambda_{2}} \cdots Q_{\beta}^{-\lambda_{\beta}}=A h(z, \xi)^{\iota_{1}} \quad \text { or } \quad H_{1}^{\mu_{1}-\frac{n_{11}}{m_{11}^{+}} \lambda_{1}} Q_{2}^{-\lambda_{2}} \cdots Q_{\beta}^{-\lambda_{\beta}}=A h(z, \xi)^{\iota_{2}}
$$

Furthermore, if $\frac{\lambda_{1}}{\mu_{1}} \geq \frac{m_{11}^{+}}{n_{11}}$, then by Corollary 4.1, we finish the proof of Theorem 4.1 by inducntion. Otherwise, we have $\frac{\lambda_{1}}{\mu_{1}}<\frac{m_{11}^{+}}{n_{11}}$, i.e. $\mu_{1}>\frac{n_{11}}{m_{11}^{+}} \lambda_{1}$. If $\operatorname{span}_{\mathbb{Q}_{0}^{+}}\left\{\mu_{1}-\frac{n_{11}}{m_{11}^{+}} \lambda_{1}\right\} \cap$ $\operatorname{span}_{\mathbb{Q}_{0}^{+}}\left\{\lambda_{2}, \cdots, \lambda_{\beta}\right\}=\{0\}$, then we are also done by the induction, as it is reduced to the $(1, \beta-1)$-factor case. Otherwise, there exist nonnegative rational numbers $d_{1}^{+}, c_{2}^{+}, \cdots, c_{\beta}^{+}$, not all zero, such that

$$
d_{1}^{+}\left(\mu_{1}-\frac{n_{11}}{m_{11}^{+}} \lambda_{1}\right)=\sum_{j=2}^{\beta} c_{j}^{+} \lambda_{j}>0
$$

Hence

$$
d_{1}^{+} \mu_{1}=d_{1}^{+} \frac{n_{11}}{m_{11}^{+}} \lambda_{1}+\sum_{j=2}^{\beta} c_{j}^{+} \lambda_{1} \neq 0 .
$$

This contradicts (1.1). This shows that $\left(H_{1}^{-n_{11}} Q_{1}^{m_{11}^{+}}\right)^{N_{0}}$ is not a rational function for any $N_{0} \in \mathbb{N}$. 
Case 1.1.2: From $H_{1}^{*} Q_{1}^{*}$ to $H_{1}^{*} Q_{1}^{*} Q_{2}^{*}$. Since $H_{1}^{-n_{11}} Q_{1}^{m_{11}^{+}}$now has a non-cyclic branching variety, applying Lemma 2.1 to (4.11), we have

$$
n_{11}^{+} \lambda_{1}=\sum_{j=2}^{\beta} n_{1 j} \lambda_{j}
$$

and

$$
h(z, \xi)^{m_{11}^{+} n_{11}^{+} r}=\left(H_{1}^{k_{22(-1)}} Q_{1}^{k_{221}} Q_{2}^{k_{222}^{+}}\right)^{-\lambda_{1}} \cdots\left(H_{1}^{k_{2 \beta(-1)}} Q_{1}^{k_{2 \beta 1}} Q_{\beta}^{k_{222}^{+}}\right)^{-\lambda_{\beta}} .
$$

Step 1.2: From $H_{1}^{*} Q_{1}^{*} \cdots Q_{p}^{*}$ to $H_{1}^{*} Q_{1}^{*} \cdots Q_{p+1}^{*}$. Now, suppose for some integer $p$ with $2 \leq$ $p \leq \beta-1$, we have

$$
n_{(p-1)(p-1)}^{+} \lambda_{p-1}=\sum_{j=p}^{\beta} n_{(p-1) j} \lambda_{j}
$$

and

$$
\begin{aligned}
h(z, \xi)^{m_{11}^{+} n_{11}^{+} \cdots n_{(p-1)(p-1)}^{+}}= & \left(H_{1}^{k_{p p(-1)}} Q_{1}^{k_{p p 1}} \cdots Q_{p-1}^{k_{p p(p-1)}} Q_{p}^{k_{p p p}^{+}}\right)^{-\lambda_{p}} \\
& \cdots\left(H_{1}^{k_{p \beta(-1)}} Q_{1}^{k_{p \beta 1}} \cdots Q_{p-1}^{k_{p v(p-1)}} Q_{\beta}^{k_{p p p}^{+}}\right)^{-\lambda_{\beta}}
\end{aligned}
$$

Case 1.2.1: Suppose

$$
H_{1}^{k_{p p(-1)}} Q_{1}^{k_{p p 1}} \cdots Q_{p-1}^{k_{p p(p-1)}} Q_{p}^{k_{p p p}^{+}}=A h(z, \xi)^{r_{4}} .
$$

We can assume $k_{p p(-1)} \leq 0$ after taking $(-1)$-th power in (4.15) if necessary. Since $k_{p p p}^{+}$may be changed to negative, we will write $k_{p p p}$ later for $k_{p p p}^{+}$. For simplicity of the notation, we assume that $k_{p p 1}, \cdots, k_{p p k}>0$ for some integer $1 \leq k \leq p$ and others are nonpositive. If there is no such $k$, it is easily reduced to the situation with $(0, p+1)$-factors and we are done by Corollary 4.1. Otherwise, by Lemma 2.3 , we can find $j^{\prime}$ from $\{-1,1, \cdots, k\}$ such that

$$
\frac{\lambda_{j}}{\lambda_{j^{\prime}}} \geq\left|\frac{k_{p p j}}{k_{p p j^{\prime}}}\right| \text { for } j=-1,1, \cdots, k \text {. }
$$

Here we write $\lambda_{-1}$ for $\mu_{1}$.

Case 1.2.1.1: Assume $j^{\prime} \neq-1$ and for simplicity of notation, assume $j^{\prime}=1$. By (4.15), one solves

$$
Q_{1}=A h(z, \xi)^{r_{5}} H_{1}^{-\frac{k_{p p(-1)}}{k_{p p 1}}} Q_{2}^{-\frac{k_{p p 2}}{k_{p p 1}}} \cdots Q_{p}^{-\frac{k_{p p p}}{k_{p p 1}}}
$$


Substituting into (4.9), we have

$$
H_{1}^{\mu_{1}+\frac{k_{p p(-1)}}{k_{p p 1}} \lambda_{1}} Q_{2}^{-\left(\lambda_{2}-\frac{k_{p p 2}}{k_{p p 1}} \lambda_{1}\right)} \cdots Q_{p}^{-\left(\lambda_{p}-\frac{k_{p p p}}{k_{p p 1}} \lambda_{1}\right)} Q_{p+1}^{-\lambda_{p+1}} \cdots Q_{\beta}^{-\lambda_{\beta}}=A h(z, \xi)^{\iota_{3}} .
$$

By (4.16), the exponent of $H_{1}$ is non-negative and all other exponents of $Q$ 's are non-positive (with some strictly negative). Now if

$$
\operatorname{span}_{\mathbb{Q}_{0}^{+}}\left\{\mu_{1}+\frac{k_{p p(-1)}}{k_{p p 1}} \lambda_{1}\right\} \cap \operatorname{span}_{\mathbb{Q}_{0}^{+}}\left\{\lambda_{2}-\frac{k_{p p 2}}{k_{p p 1}} \lambda_{1}, \cdots, \lambda_{p}-\frac{k_{p p p}}{k_{p p 1}} \lambda_{1}, \lambda_{p+1}, \cdots, \lambda_{\beta}\right\}=\{0\},
$$

we can apply the induction hypothesis to make the conclusion. Otherwise, there exist

$$
d^{+}, c_{1}, c_{2}, \cdots, c_{\beta} \in \mathbb{Z}_{0}^{+} \text {with } d^{+}>0
$$

such that

$$
d^{+} \mu_{1}=\sum_{j=2}^{\beta} c_{j} \lambda_{j}-c_{1} \lambda_{1}>0 .
$$

Here we use $\mathbb{Z}_{0}^{+}$to denote the set of non-negative integers. Also, we notice that $c_{1}>0$, for otherwise, by (1.1), we have $d^{+}=0$, which is a contradiction. Therefore, one gets

$$
c_{1} \lambda_{1}=-d^{+} \mu_{1}+\sum_{j=2}^{\beta} c_{j} \lambda_{j} .
$$

Substituting it into (4.9), we get

$$
h(z, \xi)^{c_{1} r}=\left(Q_{1}^{d^{+}} H_{1}^{c_{1}}\right)^{\mu_{1}}\left(Q_{1}^{c_{2}} Q_{2}^{c_{1}}\right)^{-\lambda_{2}} \cdots\left(Q_{1}^{c_{\beta}} Q_{\beta}^{c_{1}}\right)^{-\lambda_{\beta}} .
$$

By Lemma 2.4 and the induction hypothesis with $(1, \beta-1)$-factors, it follows that $Q_{1}^{d^{+}} H_{1}^{c_{1}}=$ $A h^{r_{6}}$. Hence $H_{1}=A_{1} h(z, \xi)^{r_{7}}$ and we finish the proof by induction.

Case 1.2.1.2: Assume $j^{\prime}=-1$. We then solve from (4.15)

$$
H_{1}=A Q_{1}^{-\frac{k_{p p 1}}{k_{p p(-1)}}} \cdots Q_{p}^{-\frac{k_{p p p}}{k_{p p(-1)}}} h(z, \xi)^{r_{8}} .
$$

Substituting it into (4.9), we get

$$
h(z, \xi)^{\iota_{4}}=A Q_{1}^{-\left(\lambda_{1}+\frac{k_{p p 1}}{k_{p p(-1)}} \mu_{1}\right)} \cdots Q_{p}^{-\left(\lambda_{p}+\frac{k_{p p p}}{k_{p p(-1)}} \mu_{1}\right)} Q_{p+1}^{-\lambda_{p+1}} \cdots Q_{\beta}^{-\lambda_{\beta}} .
$$

Notice that all exponents are non-positive by (4.16) with at least one negative. Applying Corollary 4.1, it follows that $Q_{j}=B_{j} h^{n_{j}^{+}}$. We are also done by induction. 
Case 1.2.2: Suppose $H_{1}^{k_{p p(-1)}} Q_{1}^{k_{p p 1}} \cdots Q_{p-1}^{k_{p p(p-1)}} Q_{p}^{k_{p p p}^{+}}$does not have all cyclic branching varieties. By applying Lemma 2.1 once more, we get (4.13), (4.14) with $p$ being replaced by $p+1$.

Step 1.3: By repeating the argument in Step 1.2, we will either prove the theorem for $(1, \beta)$-factor or get (4.13) for each $p \leq \beta$ and at the last stage, we have (4.14) for $p=\beta$, i.e.

$$
h(z, \xi)^{m_{11}^{+} n_{11}^{+} \cdots n_{(\beta-1)(\beta-1)}^{+}}=\left(H_{1}^{k_{\beta \beta(-1)}} Q_{1}^{k_{\beta \beta 1}} \cdots Q_{\beta}^{k_{\beta \beta \beta}^{+}}\right)^{-\lambda_{\beta}} .
$$

Hence $\frac{\lambda_{\beta}}{r} \in \mathbb{Q}$. Back to (4.13) for $2 \leq p \leq \beta$ and (4.10), it follows that $\frac{\mu_{1}}{r}, \frac{\lambda_{1}}{r}, \cdots, \frac{\lambda_{\beta}}{r}$ are all rational numbers. This is a contradiction to (1.1). This proves the $(1, \beta)$-factor case.

Hence we complete the proof for the $(1, v)$-factor case for any $v$.

Step 2: We assume that we already proved Theorem 4.1 for $(l, j)$-factors with $1 \leq l<\alpha, 1 \leq$ $j \leq \beta$. We consider the case with $(\alpha, \beta)$-factors. We have the following basic assumption:

$$
h(z, \xi)^{r}=H_{1}^{\mu_{1}} \cdots H_{\alpha}^{\mu_{\alpha}} Q_{1}^{-\lambda_{1}} \cdots Q_{\beta}^{-\lambda_{\beta}} .
$$

We can assume that $H_{1}$ has a non-cyclic branching variety. Then by applying Lemma 2.1 to (4.23), we have

$$
n_{11}^{+} \mu_{1}=\sum_{l=2}^{\alpha} n_{1 l} \mu_{l}+\sum_{j=1}^{\beta} m_{1 j} \lambda_{j}
$$

and

$$
h(z, \xi)^{n_{11}^{+} r}=\prod_{l=2}^{\alpha}\left(H_{1}^{k_{2(-l)(-1)}} H_{l}^{k_{2(-l)(-l)}^{+}}\right)^{\mu_{l}} \cdot \prod_{j=1}^{\beta}\left(H_{1}^{k_{2 j(-1)}} Q_{j}^{k_{2 j j}^{+}}\right)^{-\lambda_{j}} .
$$

Step 2.1: Suppose for $p$ with $2 \leq p \leq \alpha$, we have

$$
n_{(p-1)(p-1)}^{+} \mu_{p-1}=\sum_{l=p}^{\alpha} n_{(p-1) l} \mu_{l}+\sum_{j=1}^{\beta} m_{(p-1) j} \lambda_{j}
$$

and

$$
\begin{aligned}
h(z, \xi)^{n_{11}^{+} \cdots n_{(p-1)(p-1)}^{+}}= & \prod_{l=p}^{\alpha}\left(H_{1}^{k_{p(-l)(-1)}} \cdots H_{p-1}^{k_{p(-l)(-p+1)}} H_{l}^{k_{p(-l)(-l)}^{+}}\right)^{\mu_{l}} \\
& \cdot \prod_{j=1}^{\beta}\left(H_{1}^{k_{p j(-1)}} \cdots H_{p-1}^{k_{p j(-p+1)}} Q_{j}^{k_{p j j}^{+}}\right)^{-\lambda_{j}}
\end{aligned}
$$


Case 2.1.1: Suppose that all branching varieties of $H_{1}^{k_{p(-p)(-1)}} \cdots H_{p-1}^{k_{p(-p)(-p+1)}} H_{p}^{k_{p(-p)(-p)}^{+}}$are simple cyclic branching varieties with respect to any of its branch, then

$$
H_{1}^{k_{p(-p)(-1)}} \cdots H_{p-1}^{k_{p(-p)(-p+1)}} H_{p}^{k_{p(-p)(-p)}^{+}}=A h(z, \xi)^{r_{10}} .
$$

If $k_{p(-p)(-1)}, \cdots, k_{p(-p)(-p)}^{+} \geq 0$, then we are done by applying Corollary 4.1. We thus assume that at least one exponent is negative. For simplicity of notation, assume $k_{p(-p)(-1)}, \cdots, k_{p(-p)(-k)}$ $<0$ and $k_{p(-p)(-k-1)}, \cdots, k_{p(-p)(-p)} \geq 0$ for $1 \leq k \leq p-1$. By Lemma 2.3 , we can find $1 \leq l^{\prime} \leq k$ such that

$$
\frac{\mu_{l}}{\mu_{l^{\prime}}} \geq\left|\frac{k_{p(-p)(-l)}}{k_{p(-p)\left(-l^{\prime}\right)}}\right| \text { for } 1 \leq l \leq k
$$

We further assume $l^{\prime}=1$ to simplify the notation. Then we solve from (4.26)

$$
H_{1}=A H_{2}^{-\frac{k_{p(-p)(-2)}}{k_{p(-p)(-1)}}} \cdots H_{p}^{-\frac{k_{p(-p)(-p)}}{k_{p(-p)(-1)}}} h(z, \xi)^{r_{11}} .
$$

Substituting it into (4.23), we have

$$
H_{2}^{\mu_{2}-\frac{k_{p(-p)(-2)}}{k_{p(-p)(-1)}} \mu_{1}} \cdots H_{p}^{\mu_{p}-\frac{k_{p(-p)(-p)}}{k_{p(-p)(-1)}} \mu_{1}} H_{p+1}^{\mu_{p+1}} \cdots H_{\alpha}^{\mu_{\alpha}} Q_{1}^{-\lambda_{1}} \cdots Q_{\beta}^{-\lambda_{\beta}}=A h(z, \xi)^{\iota_{5}} .
$$

Notice that all the exponents for $H_{2}, \cdots, H_{\alpha}$ are nonnegative. Now either we are in the case of

$$
\begin{gathered}
\operatorname{span}_{\mathbb{Q}_{0}^{+}}\left\{\mu_{2}-\frac{k_{p(-p)(-2)}}{k_{p(-p)(-1)}} \mu_{1}, \cdots, \mu_{p}-\frac{k_{p(-p)(-p)}}{k_{p(-p)(-1)}} \mu_{1}, \mu_{p+1}, \cdots, \mu_{\alpha}\right\} \\
\cap \operatorname{span}_{\mathbb{Q}_{0}^{+}}\left\{\lambda_{1}, \cdots, \lambda_{\beta}\right\}=\{0\}
\end{gathered}
$$

and we then finish the proof of the theorem by the induction hypothesis, or (4.29) does not hold. Therefore, there exist $d_{1}, d_{2}, \cdots, d_{\alpha}, c_{1}, \cdots, c_{\beta} \in \mathbb{Q}_{0}^{+}$, such that

$$
\sum_{l=2}^{\alpha} d_{l} \mu_{l}-d_{1} \mu_{1}=\sum_{j=1}^{\beta} c_{j} \lambda_{j}>0 .
$$

It follows that $d_{1}>0$ by (1.1). Hence, as before, we get

$$
d_{1} \mu_{1}=\sum_{l=2}^{\alpha} d_{l} \mu_{l}-\sum_{j=1}^{\beta} c_{j} \lambda_{j} .
$$

Taking $d_{1}$-th power in (4.23) and using (4.30), it follows that 


$$
h(z, \xi)^{d_{1} r}=\prod_{l=2}^{\alpha}\left(H_{1}^{d_{l}} H_{l}^{d_{1}}\right)^{\mu_{l}} \cdot \prod_{j=1}^{\beta}\left(H_{1}^{c_{j}} Q_{j}^{d_{1}}\right)^{-\lambda_{j}} .
$$

By the same argument at the end of Case 1.2.1.1, we are done applying the induction hypothesis.

Case 2.1.2: Suppose (4.26) does not hold. By Lemma 2.1, we get (4.24), (4.25) for $p$ being replaced by $p+1$. By repeating the same argument as in Step 2.1, we will either prove the theorem, or get (4.24) for each $2 \leq p \leq \alpha+1$ and (4.25) for $p=\alpha+1$. Hence,

$$
n_{\alpha \alpha}^{+} \mu_{\alpha}=\sum_{j=1}^{\beta} m_{\alpha j} \lambda_{j}
$$

and

$$
h(z, \xi)^{n_{11}^{+} \cdots n_{\alpha \alpha}^{+} r}=\prod_{j=1}^{\beta}\left(H_{1}^{k_{(\alpha+1) j(-1)}} \cdots H_{\alpha}^{k_{(\alpha+1) j(-\alpha)}} Q_{j}^{k_{(\alpha+1) j j}^{+}}\right)^{-\lambda_{j}} .
$$

By applying Lemma 2.1, we either conclude

$$
H_{1}^{k_{(\alpha+1) 1(-1)}} \cdots H_{\alpha}^{k_{(\alpha+1) 1(-\alpha)}} Q_{1}^{k_{(\alpha+1) 11}^{+}}=A h(z, \xi)^{r_{12}}
$$

or

$$
n_{(\alpha+1)(\alpha+1)}^{+} \lambda_{1}=\sum_{l=2}^{\beta} m_{(\alpha+1) l} \lambda_{l}
$$

and

$$
h(z, \xi)^{n_{11}^{+} \cdots n_{(\alpha+1)(\alpha+1)}^{+}}=\prod_{j=2}^{\beta}\left(H_{1}^{k_{(\alpha+2) j(-1)}} \cdots H_{\alpha}^{k_{(\alpha+2) j(-\alpha)}} Q_{1}^{k_{(\alpha+2) j j}} Q_{j}^{k_{(\alpha+2) j j}^{+}}\right)^{-\lambda_{j}} .
$$

Step 2.2: Suppose we know for some integer $2 \leq p \leq \beta-1$,

$$
n_{(\alpha+p-1)(\alpha+p-1)}^{+} \lambda_{p-1}=\sum_{l=p}^{\beta} m_{(\alpha+p-1) l} \lambda_{l}
$$

and

$$
h(z, \xi)^{n_{11}^{+} \cdots n_{(\alpha+p-1)(\alpha+p-1)}^{+}}=\prod_{j=p}^{\beta}\left(H_{1}^{k_{(\alpha+p) j(-1)}} \cdots H_{\alpha}^{k_{(\alpha+p) j(-\alpha)}} Q_{1}^{k_{(\alpha+p) j 1}} \cdots Q_{j}^{k_{(\alpha+p) j j}^{+}}\right)^{-\lambda_{j}} .
$$

By applying Lemma 2.1, we either conclude 


$$
H_{1}^{k_{(\alpha+p) p(-1)}} \cdots H_{\alpha}^{k_{(\alpha+p) p(-\alpha)}} Q_{1}^{k_{(\alpha+p) p 1}} \cdots Q_{p}^{k_{(\alpha+p) p p}^{+}}=A h(z, \xi)^{r_{13}}
$$

or (4.32), (4.33) with $p$ being replaced by $p+1$.

Case 2.2.1: Assume (4.34) for $1 \leq p \leq \beta-1$. If $k_{(\alpha+p) p(-1)}=\cdots=k_{(\alpha+p) p(-\alpha)}=0$, then we are done by repeating exactly the same argument as in Case 2.1.1. Otherwise, by taking $(-1)$-th power of (4.34) if necessary, we can assume $k_{(\alpha+p) p(-1)}<0$. If this is the case, $k_{(\alpha+p) p p}^{+}$ will change the sign and hence we will write in the following $k_{(\alpha+p) p p}$ for $k_{(\alpha+p) p p}^{+}$. Suppose, without loss of generality, that there exists $1 \leq k \leq \alpha, 0 \leq k^{\prime} \leq p$, such that $k_{(\alpha+p) p(-l)}<0$ for $1 \leq l \leq k, k_{(\alpha+p) p(-l)} \geq 0$ for $l>k$, and $k_{(\alpha+p) p j}>0$ for $1 \leq j \leq k^{\prime}, k_{(\alpha+p) p j} \leq 0$ for $p \geq j>k^{\prime}$. Here $k^{\prime}=0$ means that $k_{(\alpha+p) p j} \leq 0$ for all $j=1, \cdots, p$. By Lemma 2.3, there exists $-1 \geq j^{\prime} \geq-k$ or $1 \leq j^{\prime} \leq k^{\prime}$ such that

$$
\frac{\lambda_{j}}{\lambda_{j^{\prime}}} \geq\left|\frac{k_{(\alpha+p) p j}}{k_{(\alpha+p) p j^{\prime}}}\right| \text { for } j=-1, \cdots,-k \text { and } 1, \cdots, k^{\prime},
$$

Here we use $\lambda_{j}$ to denote $\mu_{-j}$ when $j<0$.

Case 2.2.1.1: $j^{\prime}<0$. Without loss of generality and for simplicity of notation, assume $j^{\prime}=-1$. Then we solve from (4.34)

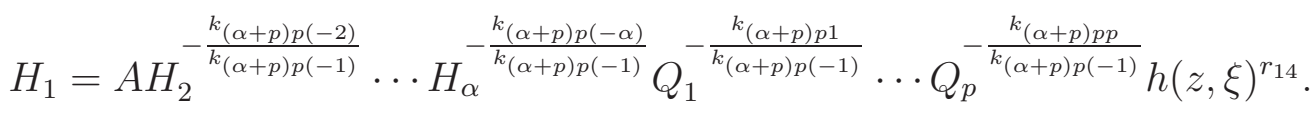

Substituting it into (4.23), we have

$$
\prod_{l=2}^{\alpha} H_{l}^{\mu_{l}-\frac{k_{(\alpha+p) p(-l)}}{k_{(\alpha+p) p(-1)}} \mu_{1}} \cdot \prod_{j=1}^{p} Q_{j}^{-\left(\lambda_{j}+\frac{k_{(\alpha+p) p j}}{k_{(\alpha+p) p(-1)}} \mu_{1}\right)} \cdot \prod_{j=p+1}^{\beta} Q_{j}^{-\lambda_{j}}=A h(z, \xi)^{\iota_{6}} .
$$

Notice that the exponents for $H_{2}, \cdots, H_{\alpha}$ are nonnegative and those of $Q_{1}, \cdots, Q_{\beta}$ are nonpositive and at least one is not zero. Now either we are in the case of

$$
\begin{aligned}
& \operatorname{span}_{\mathbb{Q}_{0}^{+}}\left\{\mu_{2}-\frac{k_{(\alpha+p) p(-2)}}{k_{(\alpha+p) p(-1)}} \mu_{1}, \cdots, \mu_{\alpha}-\frac{k_{(\alpha+p) p(-\alpha)}}{k_{(\alpha+p) p(-1)}} \mu_{1}\right\} \\
& \cap \operatorname{span}_{\mathbb{Q}_{0}^{+}}\left\{\lambda_{1}+\frac{k_{(\alpha+p) p 1}}{k_{(\alpha+p) p(-1)}} \mu_{1}, \cdots, \lambda_{p}+\frac{k_{(\alpha+p) p p}}{k_{(\alpha+p) p(-1)}} \mu_{1}, \lambda_{p+1}, \cdots, \lambda_{\beta}\right\}=\{0\}
\end{aligned}
$$

and we finish the proof of the theorem by the induction hypothesis, or (4.36) does not hold. Therefore, there exist $d_{1}, d_{2}, \cdots, d_{\alpha}, c_{1}, \cdots, c_{\beta} \in \mathbb{Z}_{0}^{+}$, such that

$$
\sum_{l=2}^{\alpha} d_{l} \mu_{l}-d_{1} \mu_{1}=\sum_{j=1}^{\beta} c_{j} \lambda_{j}>0
$$


It follows that $d_{1}>0$ by (1.1) and we finish our proof as in Case 2.1.1.

Case 2.2.1.2: $j^{\prime}>0$. Without loss of generality, assume $j^{\prime}=1$. Repeat the same argument as in Case 2.2.1.1, we also finish the proof.

Case 2.2.2: Suppose we have (4.32) (4.33) with $p$ being replaced by $p+1$. By repeating the same argument as in Case 2.2.1, we eventually have (4.32) for all $2 \leq p \leq \beta$ and (4.33) for $p=\beta$. Hence,

$$
h(z, \xi)^{n_{11}^{+} \cdots n_{(\alpha+\beta-1)(\alpha+\beta-1)^{r}}^{+}}=\left(H_{1}^{k_{(\alpha+\beta) \beta(-1)}} \cdots H_{1}^{k_{(\alpha+\beta) \beta(-\alpha)}} Q_{1}^{k_{(\alpha+\beta) \beta 1}} \cdots Q_{\beta}^{k_{(\alpha+\beta) \beta \beta}^{+}}\right)^{-\lambda_{\beta}} .
$$

Hence we conclude $\frac{\lambda_{\beta}}{r} \in \mathbb{Q}$.

Step 2.3: Since (4.32) holds for $2 \leq p \leq \beta$, (4.31) and (4.24) hold for $2 \leq p \leq \alpha$, it follows that $\frac{\mu_{1}}{r}, \cdots, \frac{\mu_{\alpha}}{r}, \frac{\lambda_{1}}{r}, \cdots, \frac{\lambda_{\beta}}{r} \in \mathbb{Q}$. We arrive at a contradiction with (1.1). This proves the $(\alpha, \beta)$-factor case.

By induction, we finally complete the proof of Theorem 4.1.

\subsection{Proof of Theorem 1.1}

As an application of Theorem 4.1, we give a proof of Theorem 1.1.

Proof of Theorem 1.1: Suppose the notation and assumption in Theorem 1.1. $F_{l}, G_{j}$ can be regarded as holomorphic maps from $U \subset \mathbb{C}^{n}$ into $\mathbb{C}^{N}$ with a large $N$, after shrinking $U$ and choosing suitable coordinates. We can assume that $F_{l}(0), G_{j}(0)=0$. Then $(1.2)$ is equivalent to the following:

$$
\sqrt{-1} \partial \bar{\partial} \log h(z, \bar{z})=\sqrt{-1} \sum_{l=1}^{m} \mu_{l} \partial \bar{\partial} \log \left(1+\left|F_{l}(z)\right|^{2}\right)-\sqrt{-1} \sum_{j=1}^{v} \lambda_{j} \partial \bar{\partial} \log \left(1+\left|G_{j}(z)\right|^{2}\right), z \in U \text {. }
$$

Taking away $\partial \bar{\partial}$ and comparing the mixed terms in the Taylor expansion of (4.37) around 0, we get (4.6). By Theorems 3.1 and 4.1, we conclude that we have $\left(1+\left|F_{l}\right|^{2}\right)=A_{l} h^{m_{l}}$ and $\left(1+\left|G_{j}\right|^{2}\right)=B_{j} h^{n_{j}}$ for each $l, j$ with $m_{l}, n_{j} \in \mathbb{N}, A_{l}, B_{j}>0$. Hence, $F_{l}:\left(U \subset M, m_{j} \omega\right) \rightarrow$ $\left(\mathbb{P}^{N}, \omega_{F S}\right)$ and $G_{j}:\left(U \subset M, n_{j} \omega\right) \rightarrow\left(\mathbb{P}^{N}, \omega_{F S}\right)$ are local isometries for all $l, j$. By the classical result of Calabi in $[\mathrm{Ca}], F_{l}, G_{j}$ extend holomorphically along any path inside $M$. Since $M$ is simply connected, we see $F_{l}$ or $G_{j}$ extend to holomorphic immersions from $M$ into $\mathbb{P}^{N_{l}}$ or $\mathbb{P}^{N_{j}^{\prime}}$, respectively, with the same kind of isometric property. The rest of the proof is easy.

We mention that Theorem 1.1 applies when $(M, \omega)$ is $\mathbb{P}^{n}$ with the standard Fubini-Study metric. Indeed, as even in the more general compact Hermitian symmetric spaces of compact type case, the extended maps are one-to-one. (See the proof of Theorem 1.2 in the following section.) 


\section{Proof of Theorem 1.2}

Let $M \subset \mathbb{P}^{n}$ be a projective algebraic manifold of complex dimension $k$. Let $\left\{U_{j}, \psi_{j}\right\}$ be a system of holomorphic charts of $M$ with $\psi_{j}: U_{j} \rightarrow V_{j} \subset \mathbb{C}^{k}$ a biholomorphic map for each $j$. (We always assume that $U_{j}^{\prime} s$ are connected.) We call $\left\{U_{j}, \psi_{j}\right\}$ is a system of Nash algebraic holomorphic charts if for each $j$ and writing $\psi_{j}^{-1}=\left[\phi_{j 0}, \cdots, \phi_{j n}\right]$ with $\phi_{j l_{0}} \not \equiv 0$, then $\frac{\phi_{j l}}{\phi_{j l_{0}}}$ is Nash algebraic over $V_{j}$ for each $l$. A holomorphic chart of $M$ is said to be a Nash algebraic holomorphic chart if the resulting transition functions with respect to $\left\{U_{j}, \psi_{j}\right\}$ are Nash algebraic. Now, a holomorphic function $h$ defined over a connected open subset $U$ of $M$ is called Nash-algebraic if for any $U_{j}$ with $U_{j} \cap U \neq \emptyset, h \circ \psi_{j}^{-1}$ is Nash algebraic over $\psi_{j}\left(U_{j} \cap U\right)$. Suppose both $M \subset \mathbb{P}^{n}$ and $M^{\prime} \subset \mathbb{P}^{n^{\prime}}$ are projective algebraic manifolds with holomorphic Nash-algebraic systems $\left\{U_{j}, \psi_{j}\right\}$ and $\left\{U_{j}^{\prime}, \psi_{j}^{\prime}\right\}$, respectively . A holomorphic map $G: U \subset M \rightarrow M^{\prime}$ is called holomorphic Nash algebraic, if $\psi_{k}^{\prime} \circ G \circ\left(\psi_{j}\right)^{-1}$ is Nash algebraic whenever it is well defined. Apparently, this definition is independent of the choice of the systems of Nash algebraic charts. Also, the Nash-algebraicity is a global property in the sense that if $G$ is Nash algebraic in a small open subset of $U$, then it is Nash algebraic over $U$.

By a variation of Chow's theorem ([p.170, GH]), when $G$ is a global map from $M$ into $M^{\prime}$, then $G$ is a restriction of a rational map from $\mathbb{P}^{n}$ into $\mathbb{P}^{n^{\prime}}$, and thus is Nash algebraic in our definition. Also, all the above definitions are independent of the embedding from the algebraic manifolds into projective spaces.

Let $\left(M, \omega_{M}\right)$ be an irreducible Hermitian symmetric space of compact type of complex dimension $n$ with a fixed canonical Kähler-Einstein metric $\omega_{M}$. We also identify $\omega_{M}$ with its associated Kähler form if there is no risk of causing confusion. By the Nakagawa-Takagi isometric embedding theorem [M1], we can assume that $M$ has been holomorphically isometrically embedded into a certain projective space $\left(\mathbb{P}^{n_{0}}, \omega_{n_{0}}\right)$ equipped with the standard Fubini-Study metric $\omega_{n_{0}}$. Let $U$ be a connected open subset of $M$. Consider local holomorphic mappings $F=\left(F_{1}, \cdots, F_{m}\right): U \rightarrow \mathbb{P}^{N_{1}} \times \cdots \times \mathbb{P}^{N_{m}}$ and $G=\left(G_{1}, \cdots, G_{v}\right): U \rightarrow \mathbb{P}^{N_{1}^{\prime}} \times \cdots \times \mathbb{P}^{N_{v}^{\prime}}$ such that

$$
\omega_{M}=\sum_{l=1}^{m} \mu_{l} F_{l}^{*} \omega_{N_{l}}-\sum_{j=1}^{v} \lambda_{j} G_{j}^{*} \omega_{N_{j}^{\prime}},
$$

where for each $j, l$, the numbers $\lambda_{j}, \mu_{l}$ are positive real numbers satisfying the number theoretic property in $(1.1)$, and $\omega_{N_{l}}, \omega_{N_{j}^{\prime}}$ are the standard Fubini-Study metric over $\mathbb{P}^{N_{l}}$ and $\mathbb{P}^{N_{j}^{\prime}}$, respectively.

Since $\left(M, \omega_{M}\right) \subset\left(\mathbb{P}^{n_{0}}, \omega_{n_{0}}\right)$ is a projective algebraic manifold, it is defined by a set of homogeneous polynomials. We can thus find a finite set of holomorphic coordinate charts $\left\{U_{i}, \Psi_{i}\right\}$, that covers $M$. We assume that each $U_{i}$ is contained in a standard complex coordinate chart of $\mathbb{P}^{n_{0}}$ and each $\Psi_{i}$ is obtained by mapping into $\mathbb{C}^{n_{0}}$ in a standard way and then projecting down to a certain open subset $V_{i}$ in the complex Euclidean space $\mathbb{C}^{n}$ with coordinates $z=$ $\left(z_{1}, \cdots, z_{n}\right)$. Write the inverse of $\Psi_{i}$ to be $\Phi_{i}=\left[\phi_{i 0}, \cdots, \phi_{i n_{0}}\right]$. Hence, there is a certain 
component $\phi_{i j} \neq 0$ over $V_{i}$. We can assume, without loss of generality, that each component of $\Phi_{i}$ is holomorphic Nash algebraic. The system of holomorphic coordinate charts thus obtained is a Nash-algebraic system. Notice that the metric $\omega_{M}$ is represented by $\sum_{\alpha, \beta=1}^{n} h_{\alpha \bar{\beta}} d z_{\alpha} \otimes d \bar{z}_{\beta}$ on $V_{i}$ with $h_{\alpha \bar{\beta}}=\frac{\partial^{2}}{\partial z_{\alpha} \partial \bar{z}_{\beta}} \log \left(\sum_{k=0}^{n_{0}}\left|\phi_{i k}\right|^{2}\right)$.

Write $\omega^{*}=\sqrt{-1} \partial \bar{\partial} \log \left(\sum_{k=0}^{n_{0}}\left|\phi_{i k}\right|^{2}\right)$. Suppose, without loss of generality, that $U \subset U_{1}$. Write $U^{*}=\Psi_{1}(U) \subset V_{1}$. Shrinking $U$ if necessary, we can further assume that for each $l, F_{l}(U)$ is contained in one of the $N_{l}+1$ standard holomorphic coordinate charts $V_{N_{l}, l_{0}}$ of $\mathbb{P}^{N_{l}}$ with the standard (linear-fractional) holomorphic coordinate map $\sigma_{N_{l}, l_{0}}: V_{N_{l}, l_{0}} \rightarrow \mathbb{C}^{N_{l}}$, and we can assume that $G_{j}(U)$ is contained in one of the $N_{j}^{\prime}+1$ standard holomorphic coordinate charts $V_{N_{j}^{\prime}, j_{0}^{\prime}}$ of $\mathbb{P}^{N_{j}^{\prime}}$ with the standard coordinate map $\sigma_{N_{j}^{\prime} j_{0}^{\prime}}: V_{N_{j}^{\prime}, j_{0}^{\prime}} \rightarrow \mathbb{C}^{N_{j}^{\prime}}$. Here, we recall that $V_{N_{j}, k}=$ $\left\{\left[z_{0}, \cdots, z_{N_{j}}\right]: z_{k} \neq 0\right\}$ and $\sigma_{N_{j}, k}\left(\left[z_{0}, \cdots, z_{N_{j}}\right]\right)=\left(z_{0} / z_{k}, \cdots, z_{k-1} / z_{k}, z_{k+1} / z_{k}, \cdots, z_{N_{j}} / z_{k}\right)$.

Proof of Theorem 1.2: Assume the notation and assumptions in Theorem 1.2 and above. As mentioned before, by the isometric embedding theorem of Nakagawa-Takagi ([Theorem 1, p.135, M1]), we need only consider maps into the product of complex projective spaces.

We now let $M$ be an irreducible Hermitian symmetric space of compact type of dimension $n$. As above, we assume that $M$ is holomorphically isometrically embedded into $\mathbb{P}^{n_{0}}$ for a certain $n_{0} \geq 2$. We let $\left(U_{1}, \Psi_{1}\right)$ be a Nash algebraic holomorphic coordinate chart of $M$ with holomorphic Nash algebraic maps

$$
\Phi_{1}=\Psi_{1}^{-1}: V_{1}=\Psi_{1}\left(U_{1}\right) \subset \mathbb{C}^{n} \rightarrow \mathbb{P}^{n_{0}}
$$

such that $U \subset U_{1}$. We also assume that $U_{1}$ is contained in one of standard coordinate charts of $\mathbb{P}^{n_{0}}$, namely, one of the coordinate in a certain fixed order never vanishes for points in $U_{1}$.

Choose a point $p^{*} \in U$ and after composing with isometries, we can assume $F_{l}\left(p^{*}\right)$ and $G_{j}\left(p^{*}\right)$ are all mapped by the standard coordinate maps to the origin in the complex Euclidean spaces. Moreover, after shrinking $U$, and thus $U^{*}:=\Psi_{1}(U)$, we can assume $F_{l}\left(U^{*}\right) \subset V_{N_{l}, l_{0}}, G_{j}\left(U^{*}\right) \subset$ $V_{N_{j}^{\prime}, j_{0}^{\prime}}$. Also, write

$$
\begin{gathered}
Y=\left(Y_{1}, \cdots, Y_{m+v}\right):= \\
\left.\left(\sigma_{N_{1}, 1_{0}} \circ F_{1} \circ \Psi_{1}^{-1}, \cdots, \sigma_{N_{m}, m_{0}} \circ F_{p} \circ \Psi_{1}^{-1}, \sigma_{N_{1}^{\prime}, 1_{0}^{\prime}} \circ G_{1} \circ \Psi_{1}^{-1}, \cdots, \sigma_{N_{v}^{\prime}, v_{0}^{\prime}} \circ G_{v} \circ \Psi_{1}^{-1}\right)\right|_{\Psi_{1}(U)},
\end{gathered}
$$

where $\sigma_{N_{j}, j_{0}}$ and $\sigma_{N_{j}^{\prime}, j_{0}^{\prime}}$ are standard coordinate maps. Then $Y\left(\Psi_{1}\left(p^{*}\right)\right)=0$.

After applying a holomorphic isometry, we also assume that $p^{*}=[1,0, \cdots, 0]$. Shrinking $U_{1}$ and $U$, we can assume that the first coordinate of points in $U_{1}$ is never zero. Write $\Psi_{1}^{-1}=$ $\left[1, \phi_{1}(z), \cdots, \phi_{n_{0}}(z)\right]$. Here, $\phi_{k}$ is algebraic and holomorphic over $V_{1}:=\Psi_{1}\left(U_{1}\right)$. Without loss of generality, we identify $p^{*}$ with the origin in $\mathbb{C}^{n_{0}}$ and $\phi_{k}(0)=0$ through the coordinate map $\Psi_{1}$. From the hypothesis (5.1), it follows that

$$
\sqrt{-1} \partial \bar{\partial} \log \left(1+\sum_{k=1}^{n_{0}}\left|\phi_{k}(z)\right|^{2}\right)+\sqrt{-1} \sum_{j=1}^{v} \lambda_{j} \partial \bar{\partial} \log \left(1+\left|G_{j}(z)\right|^{2}\right)=\sqrt{-1} \sum_{l=1}^{m} \mu_{l} \partial \bar{\partial} \log \left(1+\left|F_{l}(z)\right|^{2}\right) \text {. }
$$


Here, for simplicity of notation, we still write $F$ for $\left(\sigma_{N_{1}, 1_{0}} \circ F_{1}, \cdots, \sigma_{N_{m}, m_{0}} \circ F_{m}\right) \circ \Phi_{1}$ and $G$ for $\left(\sigma_{N_{1}^{\prime}, 1_{0}^{\prime}} \circ G_{1}, \cdots, \sigma_{N_{v}^{\prime}, v_{0}^{\prime}} \circ G_{v}\right) \circ \Phi_{1}$. As in Clozel-Ullmo [CU] and Mok [M2], (5.2) yields the following:

$$
\prod_{l=1}^{m}\left(1+\left|Y_{l}\right|^{2}\right)^{\mu_{l}} \cdot \prod_{j=1}^{v}\left(1+\left|Y_{m+j}\right|^{2}\right)^{-\lambda_{j}}=1+\sum_{k}\left|\phi_{k}(z)\right|^{2}, \quad z \in \Psi_{1}(U) .
$$

Now, by Theorem 3.1, we know that $Y$ is algebraic over $V_{1}$.

We next prove that $Y$ admits a holomorphic extension along any path in $V_{1}$. Suppose not. We will have an irreducible branching variety $E$ for (some component of) $Y$ in $V_{1}$. Let $p_{0}^{*} \in E$ be a generic smooth point. Then after an algebraic holomorphic change of coordinates, we can assume that $p_{0}^{*}=0$ and $E$ near 0 is defined by $z_{n}=0$. Now for $p^{*}$ (near 0$) \notin E$, we have a certain branch $\widehat{Y}$ of $Y$ near $p^{*}$, which extends to a multi-valued holomorphic map in a small neighborhood of 0 and admits the following Puiseux expansion:

$$
\widehat{Y}=\sum_{\alpha=0}^{\infty} \tilde{a}_{\alpha}\left(z^{\prime}\right) z_{n}^{\frac{\alpha}{N}}
$$

Here we can find the smallest positive integer $\alpha_{0}$ with $N \nmid \alpha_{0}$ such that the vector-valued $\tilde{a}_{\alpha_{0}}\left(z^{\prime}\right) \not \equiv 0$ for $z^{\prime}$ near 0 . By the uniqueness of real analytic functions, we still have (5.3) near $p^{*}$. Now, for any $z_{0}^{\prime}$ near 0 , we can find a minimal rational curve $C_{z_{0}^{\prime}}$ passing through $\Psi_{1}^{-1}\left(z_{0}^{\prime}, 0\right)$ such that the tangent of $\Psi_{1}\left(C_{z_{0}^{\prime}}\right)$ at $\left(z_{0}^{\prime}, 0\right)$ is transversal to $E$ at 0 . (This is due to the fact that for any $q \in M$, the holomorphic (isometric) isotropic subgroup acts irreducibly on $T_{q}^{(1,0)}$ and the subspace of the span of the tangent vectors of minimal rational curves at $q$ is an invariant subspace of $T_{q}^{(1,0)}$.) Write a holomorphic parametrization of $\Psi_{l}\left(C_{z_{0}^{\prime}}\right)$ as: $z^{\prime}=\phi^{\prime}(\eta), z_{n}=\phi_{n}(\eta)$ with $\phi^{\prime}(0)=z_{0}^{\prime}, \phi_{n}(0)=0$ and $\phi_{n}(\eta)=\eta h(\eta)$ with $h(0) \neq 0$. Restrict $Y^{*}$ to $\Psi_{1}\left(C_{z_{0}^{\prime}}\right)$ near $\left(z_{0}^{\prime \prime}, z_{n}^{\prime \prime}\right)$ with certain $z_{n}^{\prime \prime}(\neq 0) \approx 0$ and $z_{0}^{\prime \prime} \approx z_{0}^{\prime}$. Let $\omega_{C_{z_{0}^{\prime}}}=\left.\omega_{M}\right|_{C_{z_{0}^{\prime}}}$. Then we know that $\left(C_{z_{0}^{\prime}}, \omega_{z_{z_{0}^{\prime}}}\right)$ is isometric to $\left(\mathbb{P}^{1}, \omega_{1}\right)$. Now, by Theorem 1.1 with $M=\mathbb{P}^{1}$, we see that $F, G$ admit a global holomorphic extension to $C_{z_{0}^{\prime}}$. Hence,

$$
\tilde{a}_{\alpha_{0}}\left(\phi^{\prime}(\eta)\right) h(\eta)^{\frac{\alpha_{0}}{N}} \equiv 0 \quad \text { for } \quad \eta \text { near } 0 .
$$

In particular, $\tilde{a}_{\alpha_{0}}\left(z_{0}^{\prime}\right)=0$. Since $z_{0}^{\prime}$ is arbitrary, we see that $\tilde{a}_{\alpha_{0}}\left(z^{\prime}\right) \equiv 0$. This is a contradiction.

We next claim that $F$ (resp. $G$ ) admits a holomorphic extension along any path $\gamma \subset M$ with $\gamma(0) \in U$. Indeed, if not, we can find $t_{0} \in(0,1]$ such that $F$ (resp. $G$ ) admits a holomorphic extension along $\left.\gamma\right|_{\left[0, t^{\prime}\right]}$ for any $t^{\prime}<t_{0}$ but not along $\left.\gamma\right|_{\left[0, t_{0}\right]}$. Assume that $\gamma\left(t_{0}\right) \in U_{i_{0}}$ for some $i_{0}$, where $\left(U_{i_{0}}, \Psi_{i_{0}}\right)$ is a Nash algebraic holomorphic chart of $M$ with the similar property as described for $\left(U_{1}, \Psi_{1}\right)$. Then $\gamma\left(t^{\prime}\right) \in U_{i_{0}}$ for $t^{\prime}\left(<t_{0}\right)$ sufficiently close to $t_{0}$. Composing with a holomorphic isometry of $\mathbb{P}^{n_{0}}$, we can make $p^{*}:=\gamma\left(t^{\prime}\right)=[1,0, \cdots, 0]$. Also, when $t^{\prime}$ is sufficiently close to $t_{0}$, we can assume that a small neighborhood, still denoted by $U_{i_{0}}$, of $\gamma\left(t^{\prime}\right)$ contains $\gamma\left(t_{0}\right)$. Also, we still have a Nash holomorphic coordinate map $\Psi_{i_{0}}$ over $U_{i_{0}}$. Notice that 
composing $F$ or $G$ on the right by automorphisms of the target manifold will never change the holomorphic extendability. Now composing $F_{l}$ and $G_{j}$ by holomorphic isometres, we can assume that $F_{l}\left(p^{*}\right)=[1,0, \cdots, 0]$ and $G_{j}\left(p^{*}\right)=[1,0, \cdots, 0]$. Hence, repeating exactly the argument as above, it follows that the map obtained by restricting the extended map $F$ (resp. $G$ ) to a small neighborhood of $\gamma\left(t^{\prime}\right)$ admits a holomorphic extension along curves in $U_{i_{0}}$. In particular, we conclude that $F$ and $G$ extend holomorphically along $\gamma\left(\left[0, t_{0}\right]\right)$. This is a contradiction. Now, since $M$ is simply connected, we conclude that $F$ (resp. $G$ ) extends to a holomorphic map $\tilde{F}$ (resp. $\tilde{G})$ from $M$ into $\mathbb{P}^{N_{1}} \times \cdots \times \mathbb{P}^{N_{m}}$ (resp. $\left.\mathbb{P}^{N_{1}^{\prime}} \times \cdots \times \mathbb{P}^{N_{v}^{\prime}}\right)$.

Finally, we show that each $\tilde{F}_{l}$ (resp. $\tilde{G}_{j}$ ) is a holomorphic isometric embedding from $M$ into $\mathbb{P}^{N_{l}}$ (resp. $\mathbb{P}^{N_{j}^{\prime}}$ ) up to a certain isometric constant $m_{l}\left(\right.$ resp. $\left.n_{j}\right) \in \mathbb{N}$. Theorem $1.1, \tilde{F}_{l}$, when restricted to each minimal rational curve $C$, is a holomorphic isometric embedding up to an isometric constant. Notice that $\tilde{F}_{l}^{*}\left(\frac{1}{2 \pi} \omega_{N_{l}}\right)$ is a closed $(1,1)$-form on $M$, which is also an element in $H^{2}\left(M_{l}, \mathbb{Z}\right)$. Since $H^{2}(M, \mathbb{Z})$ is generated by $\frac{1}{2 \pi} \omega_{M}$, we have a certain $m_{l} \in \mathbb{Z}$ such that

$$
\tilde{F}_{l}^{*} \omega_{N_{l}}-m_{l} \omega_{M}=\sqrt{-1} \partial \bar{\partial} \vartheta
$$

for a certain real-valued, real analytic function $\vartheta$ over $M$. Restricting to each minimal rational curve $C$, we get

$$
\left.\sqrt{-1} \partial \bar{\partial} \vartheta\right|_{C}=m_{C} \omega_{C}
$$

Since $\omega_{C}$ is positive definite, we see that $\left.\vartheta\right|_{C}$ is either subharmonic or superharmonic over $C$. Thus $\vartheta$ is constant on $C$ as $C$ is compact. Since any two points in $M$ can be connected by a finite sequence of minimal rational curves (see $[\mathrm{HK}]$ ), we see that $\vartheta$ is a constant over $M$. Hence we have

$$
\tilde{F}_{l}^{*} \omega_{N_{l}}=m_{l} \omega_{M}
$$

Since $F_{l}$ is not constant, we see that $m_{l}>0$. Now, applying the Nakagawa-Takagi theorem and applying the local uniqueness theorem (up to isometries) of Calabi ([Theorem 9, C]), we see that $F_{l}$ (resp. $G_{j}$ ) coincides with the (one-to-one) $m_{l}$-th (resp. $n_{j}$-th) cannonical embedding from $M$ into $M_{l}$ (resp. $M_{j}^{\prime}$ ) upto a unitary action. The identity (1.3) thus also holds. The proof of Theorem 1.2 is complete.

\section{Examples and Remarks}

Remark 6.1 (Calabi $[\mathrm{p} .23, \mathrm{C}]):\left(\mathbb{P}^{n}, \mu \omega_{n}\right)$ can be locally holomorphically and isometrically embedded into $\left(\mathbb{P}^{\infty}, \lambda \omega_{\infty}\right)$ if and only if $\lambda=k \mu$ with $k \in \mathbb{N}$. This fact can be easily seen to be equivalent to the following simple statement: There is a sequence of holomorphic functions $\left\{f_{i}(z)\right\}_{i=1}^{\infty}$ defined in a certain fixed small neighborhood of $0 \in \mathbb{C}^{n}$ with $f_{i}(0)=0$ for each $i$ 
such that $\left(1+|z|^{2}\right)^{\lambda / \mu}=1+\sum_{i=1}^{\infty}\left|f_{i}(z)\right|^{2}$ if and only if $\lambda / \mu$ is a positive integer. (When $\lambda / \mu$ is not a positive integer, many coefficients for terms of the form $|z|^{2 k}$ in the Taylor expansion of the left hand side are negative, while such kind of terms in the right hand side have nonnegative coefficients.) Hence, as in Calabi's paper [p.23, C], if $\mu_{1} / \mu_{2}$ is not rational, there is no $\lambda$ such that both $\left(\mathbb{P}^{n}, \mu_{1} \omega_{n}\right)$ and $\left(\mathbb{P}^{m}, \mu_{2} \omega_{m}\right)$ can be (locally holomorphically and isometrically) embedded into $\left(\mathbb{P}^{\infty}, \lambda \omega_{\infty}\right)$. Thus $\left(\mathbb{P}^{n} \times \mathbb{P}^{m}, \mu_{1} \omega_{n} \oplus \mu_{2} \omega_{m}\right)$ can not be locally holomorphically and isometrically embedded into $\left(\mathbb{P}^{\infty}, \lambda \omega_{\infty}\right)$ for any choice of $\lambda$.

Example 6.2: Let $\left\{\mu_{1}, \cdots, \mu_{m}\right\}$ and $\left\{\lambda_{1}, \cdots, \lambda_{v}\right\}$ be two sets of positive numbers. Suppose that there exist nonnegative integers $m_{l}^{\prime}, n_{j}^{\prime}$ such that

$$
\sum_{l=1}^{m} m_{l}^{\prime} \mu_{l}=\sum_{j=1}^{v} n_{j}^{\prime} \lambda_{j}>0 .
$$

Now, if there is a holomorphic map $G=\left(G_{1}, \cdots, G_{v}\right):\left(\mathbb{C}^{n} \subset \mathbb{P}^{n}, \omega_{n}\right) \rightarrow\left(\mathbb{P}^{N_{1}^{\prime}} \times \cdots \times\right.$ $\left.\mathbb{P}^{N_{v}^{\prime}}, \oplus_{j=1}^{v} \lambda_{j} \omega_{N_{j}^{\prime}}\right)$ and holomorphic map $F=\left(F_{1}, \cdots, F_{m}\right):\left(\mathbb{C}^{n} \subset \mathbb{P}^{n}, \omega_{n}\right) \rightarrow\left(\mathbb{P}^{N_{1}} \times \cdots \times\right.$ $\left.\mathbb{P}^{N_{m}}, \oplus_{l=1}^{m} \mu_{l} \omega_{N_{l}}\right)$ such that $\omega_{\mathbb{P}^{n}, G, \lambda}=\sum_{l=1}^{m} \mu_{l} F_{l}^{*} \omega_{N_{l}}$, and each mapping factor is itself an isometry up to a conformal factor, then we see that there are positive integers $m_{l}, n_{j}$ such that

$$
\sum_{l=1}^{m} m_{l} \mu_{l}=\sum_{j=1}^{v} n_{j} \lambda_{j}+1
$$

On the other hand, assume (6.1). Given any holomorphic map $f: 0 \in U \subset \mathbb{C}^{n} \rightarrow \mathbb{C}^{N}$ with $f(0)=0$, define

$$
h_{l}=\left(1+|z|^{2}\right)^{m_{l}}\left(1+|f|^{2}\right)^{m_{l}^{\prime}} \text { and } q_{j}=\left(1+|z|^{2}\right)^{n_{j}}\left(1+|f|^{2}\right)^{n_{j}^{\prime}}
$$

Then

$$
h_{1}^{\mu_{1}} \cdots h_{m}^{\mu_{m}} q_{1}^{-\lambda_{1}} \cdots q_{v}^{-\lambda_{v}}=1+|z|^{2} .
$$

By Lemma 2.4, one can construct holomorphic maps $F_{l}: U \subset \mathbb{C}^{n} \subset \mathbb{P}^{n} \rightarrow \mathbb{P}^{N_{l}}$ and $G_{j}: U \subset$ $\mathbb{C}^{n} \subset \mathbb{P}^{n} \rightarrow \mathbb{P}^{N_{j}^{\prime}}$ such that $\left(1+\left|F_{l}\right|^{2}\right)=\left(1+|z|^{2}\right)^{m_{l}}\left(1+|f|^{2}\right)^{m_{l}^{\prime}},\left(1+\left|G_{j}\right|^{2}\right)=\left(1+|z|^{2}\right)^{n_{j}}\left(1+|f|^{2}\right)^{n_{j}^{\prime}}$. Hence, by (6.2), we have

$$
\sum_{l=1}^{m} \mu_{l} F^{*} \omega_{N_{l}}=\sum_{j=1}^{v} \lambda_{j} G^{*} \omega_{N_{j}^{\prime}}+\omega_{n} .
$$

Notice that $f$ will be merged as part of the components of $F$ and $G$. Since $f$ is arbitrarily assigned, we do not have algebraicity, global extendability and rigidity for $F$ and $G$.

On the other hand, it is easy to see that when $\left\{\mu_{l}\right\}\left\{\lambda_{j}\right\}$ satisfy (1.1) and (1.3), for each Hermitian symmetric space of compact type $\left(M, \omega_{M}\right)$, we can find $F_{l}:\left(M, \omega_{m}\right) \rightarrow\left(\mathbb{P}^{N_{l}}, \omega_{N_{l}}\right)$ and $G_{j}:\left(M, \omega_{m}\right) \rightarrow\left(\mathbb{P}^{N_{j}^{\prime}}, \omega_{N_{j}^{\prime}}\right)$ such that $F_{l}^{*} \omega_{N_{l}}=m_{l} \omega_{M}, G_{j}^{*} \omega_{N_{j}^{\prime}}=n_{j} \omega_{M}$, and $\omega_{M, G, \lambda}=$ $\sum_{l=1}^{m} \mu_{l} F_{l}^{*} \omega_{M_{l}}$. 
Finally, one can easily construct many examples of $\lambda_{j}$ and $\mu_{l}$ such that both (1.1) and (1.3) hold. One simple example is given as follows: Let $v=1, m=2, \lambda_{1}=\sqrt{2},\left(\mu_{1}, \mu_{2}\right)=\left(\sqrt{2}+\frac{1}{4}, \frac{1}{4}\right)$. Then (1.1) holds trivially. Meanwhile, $2 \mu_{1}+2 \mu_{2}=2 \lambda_{1}+1$. Also, notice that $\mu_{2} / \mu_{1}$ is irrational and thus $\left(\mathbb{P}^{N_{1}} \times \mathbb{P}^{N_{2}}, \mu_{1} \omega_{N_{1}} \oplus \mu_{2} \omega_{N_{2}}\right)$ can not be embedded into $\left(\mathbb{P}^{\infty}, \mu \omega_{\infty}\right)$ for any $\mu>0$.

Example 6.3: Let $\left\{\mu_{l}\right\}_{l=1}^{m},\left\{\lambda_{j}\right\}_{j=1}^{v}$ be two sets of positive real numbers and let $\left\{m_{l}\right\}_{l=1}^{m},\left\{n_{j}\right\}_{j=1}^{v}$ be two sets of positive nature numbers such that (1.3) holds. Then for any irreducible Hermitian symmetric space $\left(M, \omega_{M}\right)$ of compact type, equipped with a Kähler-Einstein metric $\omega_{M}$ normalized as in Theorem 1.2, by the Nakagawa-Takagi theorem [M1], there is a holomorphic isometric embedding $F_{l}$ (resp. $G_{j}$ ) from $\left(M, \omega_{M}\right)$ into $\left(\mathbb{P}^{N_{l}}, \omega_{N_{l}}\right)$ (resp. $\left.\left(\mathbb{P}^{N_{j}^{\prime}}, \omega_{N_{j}^{\prime}}\right)\right)$ with $\omega_{N_{l}}\left(\right.$ resp. $\left.\omega_{N_{j}^{\prime}}\right)$ the standard Fubini-Study metric such that $F_{l}^{*} \omega_{N_{l}}=m_{l} \omega_{M}$ (resp. $G_{j}^{*} \omega_{N_{j}^{\prime}}=n_{j} \omega_{M}$ ). Thus, $F=\left(F_{1}, \cdots, F_{m}\right):\left(M, \omega_{M}+\sum_{j=1}^{v} \lambda_{j} G_{j}^{*} \omega_{N_{j}^{\prime}}\right) \rightarrow\left(\mathbb{P}^{N_{1}} \times \cdots \times \mathbb{P}^{N_{m}}, \oplus_{l=1}^{m} \mu_{l} \omega_{N_{l}}\right)$ is an isometric embedding.

\section{References}

[BH] M. S. Baouendi, X. Huang, Super-rigidity for holomorphic mappings between hyperquadrics with positive signature, J. Differential Geom. 69 (2005), no. 2, 379-398.

[C] E. Calabi, Isometric imbedding of complex manifolds, Ann. of Math. (2) 58, (1953). 1-23.

[CU] L. Clozel, E. Ullmo, Correspondances modulaires et mesures invariantes, J. Reine Angew. Math. 558 (2003), 47-83.

[DL] A. Di Scala, A. Loi, Kähler maps of Hermitian symmetric spaces into complex space forms, Geom. Dedicata 125 (2007), 103-113.

[GH] P. Griffiths, J. Harris, Principles of algebraic geometry, Pure and Applied Mathematics, Wiley-Interscience, New York, 1978.

[Hu1] X. Huang, On the mapping problem for algebraic strongly pseudoconvex hypersurfaces in complex spaces of different dimensions, Ann. Inst. Fourier (Grenoble) 44 (1994), no. 2, 433-463.

[Hu2] X. Huang, On a linearity problem for proper holomorphic maps between balls in complex spaces of different dimensions, J. Differential Geom. 51 (1999), no. 1, 1333.

[Hw] J.-M. Hwang, Geometry of minimal rational curves on Fano manifolds, School on Vanishing Theorems and Effective Results in Algebraic Geometry (Trieste, 2000), 335-393, ICTP Lect. Notes, 6, Abdus Salam Int. Cent. Theoret. Phys., Trieste, 2001.

[HK] J.-M. Hwang, S. Kebekus, Geometry of chains of minimal rational curves, J. Reine Angew. Math. 584 (2005), 173-194. 
[M1] N. Mok, Metric rigidity theorems on Hermitian locally symmetric manifolds, Series in Pure Mathematics. 6. World Scientific Publishing Co., Inc., Teaneck, NJ, 1989. xiv+278 pp.

[M2] N. Mok, Local holomorphic isometric embeddings arising from correspondences in the rank-1 case, Contemporary trends in algebraic geometry and algebraic topology (Tianjin, 2000), 155-165, Nankai Tracts Math. 5, World Sci. Publ., River Edge, NJ, 2002.

[M3] N. Mok, Extension of germs of holomorphic isometries up to normalizing constants with respect to the Bergman metric, J. Eur. Math. Soc. (JEMS) 14 (2012), no. 5, 1617-1656.

[M4] N. Mok, Geometry of holomorphic isometries and related maps between bounded domains, Geometry and analysis. No. 2, 225-270, Adv. Lect. Math. (ALM), 18, Int. Press, Somerville, MA, 2011.

[M5] N. Mok, Private communications, 2011.

[MN] N. Mok, S.-C. Ng, Germs of measure-preserving holomorphic maps from bounded symmetric domains to their Cartesian products, J. Reine Angew. Math. 669 (2012), 47-73.

[N1] S.-C. Ng, On holomorphic isometric embeddings of the unit disk into polydisks, Proc. Amer. Math. Soc. 138 (2010), no. 8, 2907-2922.

[N2] S.-C. Ng, On holomorphic isometric embeddings of the unit n-ball into products of two unit m-balls, Math. Z. 268 (2011), no. 1-2, 347-354.

[U] M. Umehara, Einstein Kähler submanifolds of a complex linear or hyperbolic space, Tohoku Math. J. (2) 39 (1987), no. 3, 385-389.

[Y] Y. Yuan, Ph.D. thesis at Rutgers University at New Brunswick, 2010.

[YZ] Y. Yuan, Y. Zhang, Rigidity for local holomorphic conformal embeddings from $\mathbb{B}^{n}$ into $\mathbb{B}^{N_{1}} \times \cdots \times \mathbb{B}^{N_{m}}$, J. Differential Geom. 90 (2012), no. 2, 329-349.

* Department of Mathematics, Rutgers University, New Brunswick, NJ 08903, USA

${ }^{\dagger}$ Department of Mathematics, Johns Hopkins University, Baltimore, MD 21218, USA 